Article

\title{
Nonintrusive Finger-Vein Recognition System Using NIR Image Sensor and Accuracy Analyses According to Various Factors
}

\author{
Tuyen Danh Pham, Young Ho Park, Dat Tien Nguyen, Seung Yong Kwon and Kang Ryoung Park * \\ Division of Electronics and Electrical Engineering, Dongguk University, 26 Pil-dong 3-ga, \\ Jung-gu, Seoul 100-715, Korea; E-Mails: phamdanhtuyen@gmail.com (T.D.P.); \\ fdsarew@dongguk.edu (Y.H.P.);nguyentiendat@dongguk.edu (D.T.N.); sbaru07@dgu.edu (S.Y.K.) \\ * Author to whom correspondence should be addressed; E-Mail: parkgr@dongguk.edu; \\ Tel.: +82-10-3111-7022; Fax: +82-2-2277-8735.
}

Academic Editor: Gonzalo Pajares Martinsanz

Received: 1 May 2015 / Accepted: 9 July 2015 / Published: 13 July 2015

\begin{abstract}
Biometrics is a technology that enables an individual person to be identified based on human physiological and behavioral characteristics. Among biometrics technologies, face recognition has been widely used because of its advantages in terms of convenience and non-contact operation. However, its performance is affected by factors such as variation in the illumination, facial expression, and head pose. Therefore, fingerprint and iris recognitions are preferred alternatives. However, the performance of the former can be adversely affected by the skin condition, including scarring and dryness. In addition, the latter has the disadvantages of high cost, large system size, and inconvenience to the user, who has to align their eyes with the iris camera. In an attempt to overcome these problems, finger-vein recognition has been vigorously researched, but an analysis of its accuracies according to various factors has not received much attention. Therefore, we propose a nonintrusive finger-vein recognition system using a near infrared (NIR) image sensor and analyze its accuracies considering various factors. The experimental results obtained with three databases showed that our system can be operated in real applications with high accuracy; and the dissimilarity of the finger-veins of different people is larger than that of the finger types and hands.
\end{abstract}

Keywords: nonintrusive finger-vein capturing device using NIR image sensor; misalignment of finger-vein image; multiple images for enrollment; score-level fusion 


\section{Introduction}

Recent developments have led to the widespread use of biometric technologies, such as face, fingerprint, vein, iris, and voice recognition, in a variety of applications in access control, financial transactions on mobile devices, and automatic teller machines (ATMs) [1-4]. Among them, finger-vein recognition has been highlighted because it can overcome several drawbacks of other biometric technologies, such as the effect of sweat, skin distortions, and scars in fingerprint recognition, or the effect of poses and illumination changes in face recognition. Moreover, a finger-vein recognition system is cost effective in comparison, and offers high accuracy together with the advantages of fake detection and a bio-cryptography system [5]. Finger-vein recognition uses the vascular patterns inside human fingers to uniquely identify individuals. Vein imaging technology relies on the use of near infrared (NIR) illuminators at a wavelength longer than about $750 \mathrm{~nm}$, because the deoxyhemoglobin in veins absorbs light in this range [6,7]. Previous work on finger-vein recognition include research aimed at enhancing vein image quality, increasing recognition accuracy by various feature extraction methods, considering finger veins as a factor for individual recognition in multimodal systems, as well as detecting fake finger veins. The research on finger-vein image enhancement, which is based on a software algorithm, can be classified into restoration-based and non-restoration-based methods $[7,8]$. The restoration-based methods proposed by Yang et al. [9-11] were able to produce enhanced finger-vein images by considering the effect of the layered structure of skin and restored the images by using a point-spread function (PSF) model [10], and a biological optical model (BOM) [11]. In the non-restoration-based approaches, Gabor filtering was popularly used [6-8,12,13]. Yang et al. introduced an enhancement method that uses multi-channel even-symmetric Gabor filters with four directions to strengthen the vein information in different orientations [6]. A study by Park et al. [8] led to the proposal of an image enhancement method using an optimal Gabor filter based on the directions and thickness of the vein line. An adaptive version of the Gabor filter was used in the research of Cho et al. [12] to enhance the distinctiveness of the finger-vein region in the original image. The Gabor filter was also used in combination with a Retinex filter, by using fuzzy rules in the method proposed by Shin et al. [7]. Zhang et al. proposed gray-level grouping (GLG) for the enhancement of image contrast, and a circular Gabor filter (CGF) for the enhancement of finger-vein images [13].

Pi et al. proposed a quality improvement method based on edge-preserving and elliptical high-pass filters capable of maintaining the edges and removing blur [14]. In addition, Yu et al. proposed a fuzzy-based multi-threshold algorithm considering the characteristics of the vein patterns and skin region [15].

Work has also been conducted on extracting and combining various features from finger-vein images to increase the quality of the recognition results [16-19]. In [16], they used both the global feature of the moment-invariants method and Gabor filter-based local features. In the method proposed by Lu et al. [17], eight-channel Gabor features were extracted and analyzed prior to application to score-level fusion to obtain the final matching score. Qian et al. [18] proposed a finger-vein recognition algorithm based on the fusion of score level moment invariants by the weighted-average method. In [19], Yang et al. proposed a binary feature for finger-vein matching, termed personalized best bit map (PBBM), which was extracted based on the consistent bits in local binary pattern (LBP) codes. Finger-vein recognition was also considered as a sub-system in multimodal biometric systems [20-23] along with other individual recognition methods 
to compensate for the drawbacks of each of the recognition methods. The results of finger-vein and fingerprint recognitions were matched and combined by using various methods, such as decision level fusion of "AND" or "OR" rules as in [20], a support vector machine (SVM) as in [21], or score level fusion as in [22]. He et al. [23] proposed a multimodal biometric system by considering the three biometric characteristics of fingerprint, face, and finger-vein, and evaluated the performance of the system with the use of sum rule-based and SVM-based score level fusion. The research on finger-vein recognition has also taken counterfeit vein information into account, as in [24,25]. In the anti-spoofing system for vein identification in [24], live fingers were detected by continuously capturing successive heart-rate-based images and then examining the details in the series of images. Nguyen et al. [25] proposed an image-analysis method for fake finger-vein detection based on Fourier transform and wavelet transforms.

A number of research efforts on finger-vein recognition have considered the quality of the preprocessed images, as well as the effectiveness of the matching features. However, the evaluation of the discriminant factors on finger-vein information, such as the differences between people, left and right hands, and the type of finger, has not received much attention. In our research, we propose a nonintrusive finger-vein capturing device.

Table 1. Comparison of the proposed method with previous methods.

\begin{tabular}{|c|c|c|c|}
\hline Category & Methods & Strengths & Weaknesses \\
\hline $\begin{array}{l}\text { Accuracy evaluation } \\
\text { without considering the } \\
\text { various factors of } \\
\text { people, hands, finger } \\
\text { types, and the number } \\
\text { of images }\end{array}$ & $\begin{array}{l}\text { EER or ROC curve-based } \\
\text { evaluation of finger-vein } \\
\text { recognition with the assumption that } \\
\text { the veins from different hands or } \\
\text { finger types are different classes } \\
\text { without comparing the dissimilarity } \\
\text { of finger-vein among people, hands, } \\
\text { and finger types [7-9,11,16-23,25] }\end{array}$ & $\begin{array}{l}\text { New methods for enhancing } \\
\text { finger-vein images with feature } \\
\text { extraction or score fusions for } \\
\text { enhancing the recognition } \\
\text { accuracy are proposed }\end{array}$ & $\begin{array}{l}\text { Assuming the veins from } \\
\text { different hands or finger types } \\
\text { are different classes without any } \\
\text { theoretical or experimental } \\
\text { ground }\end{array}$ \\
\hline $\begin{array}{l}\text { Accuracy evaluation } \\
\text { according to people, } \\
\text { hands, finger types, and } \\
\text { the number of images }\end{array}$ & $\begin{array}{l}\text { The dissimilarity of finger-veins } \\
\text { among people, hands, and finger } \\
\text { types are quantitatively evaluated } \\
\text { (Proposed method) }\end{array}$ & $\begin{array}{l}\text { Providing the experimental } \\
\text { ground for the dissimilarity of } \\
\text { finger-veins among people, } \\
\text { hands, and finger types }\end{array}$ & $\begin{array}{l}\text { Not providing the experimental } \\
\text { ground for the dissimilarity of } \\
\text { palm-veins or hand dorsal veins } \\
\text { among people and hands }\end{array}$ \\
\hline
\end{tabular}

Our research is novel in the following three ways compared to previous work.

- We propose a nonintrusive finger-vein capturing device using a small-sized web-camera and NIR light-emitting diodes. To reduce the misalignment of captured images while ensuring minimal user inconvenience, two guiding bars for positioning the fingertip and side of the finger were attached to the device.

- The accuracies of recognition were compared by assuming that images from the same person, the same hand, and the same finger types form the same classes. Based on the receiver operational characteristic curve, equal error rate, authentic and imposter matching distributions, and d-prime value, the dissimilarity of finger-veins among people, hands, and finger types are quantitatively evaluated.

- The accuracies of recognition are compared according to the number of finger-vein images combined by score-level fusion for recognition, and the number of images for enrollment. 
Table 1 presents a comparison of the proposed method with previous methods.

The remainder of this paper is organized as follows. Section 2, explains the details of the proposed method and Section 3, shows the experimental results and discussions. Finally, the conclusions and opportunities for future work are given in Section 4.

\section{Finger-Vein Recognition and Evaluation Method}

\subsection{Overview of the Finger-Vein Recognition System}

An overview of the proposed method is shown in Figure 1. Because the input finger-vein image consists of two parts, i.e., the finger region containing the finger-vein information and the background region, the method to detect the finger region is first applied in order to remove the background, which contains unnecessary information. In the next step, based on the detected upper and lower finger boundaries detected in the previous step, the segmented finger region is stretched into a rectangular form in the normalization step. The processing time is reduced by obtaining a sub-sample of the stretched finger-vein image to reduce the size of the image. Before the recognition features are extracted, the quality of the finger-vein image is enhanced by using Gabor filtering, subsequent to which the preprocessed image is applied to the feature extraction step using the local binary pattern (LBP) method. In the next step, the hamming distance (HD) is calculated to determine the matching distance between the extracted code features of the input finger-vein image and the enrolled image. The input finger-vein image is then classified as either being genuine or being that of an imposter by using the enrolled data based on the matching distance.

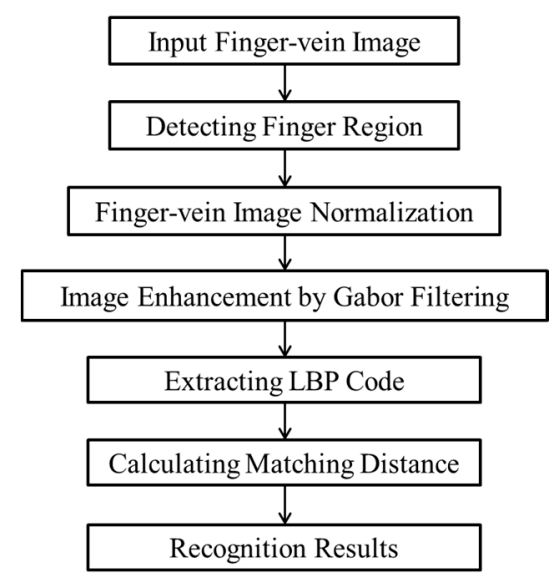

Figure 1. Flowchart of the experimental procedure of our research.

\subsection{Finger Region Detection and Normalization}

As shown in Figure 2, a captured finger-vein image consists of the background surrounding the finger region, which contains the vein pattern, which is used for recognition purposes, and which has higher gray levels than the background. The background is removed from the captured image by detecting the four boundaries of the finger region consisting of the left and right boundaries in the horizontal direction, and upper and lower boundaries in the vertical direction, based on previous research [7]. In the images from the three databases, the left and right finger region boundaries are restricted by the size of the hole 
in the device for capturing the finger-vein image. Detailed explanations of the three databases and the device are provided in Section 3. As such, the values of $X_{L}$ and $X_{R}$, which determine the left and right boundaries, as shown in Figure 2, are experimentally defined for the three databases. In the case of the good-quality database with $640 \times 480$ pixel images, the values of $X_{L}$ and $X_{R}$ are 180 and 480 pixels, respectively. For the mid-quality database with the same image size, the values of $X_{L}$ and $X_{R}$ are 220 and 470 pixels, respectively. The third (open) database, which consists of images with a size of $320 \times 240$ pixels, $X_{L}$ and $X_{R}$ are 20 and 268 pixels, respectively.

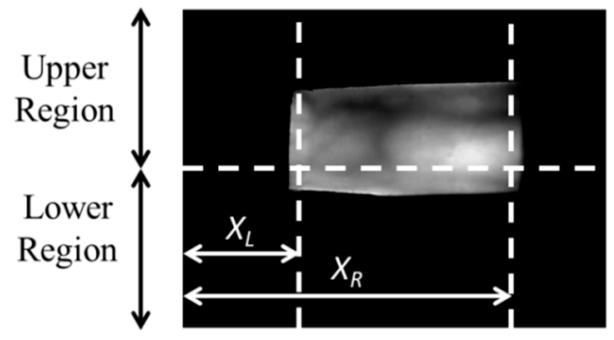

(a)

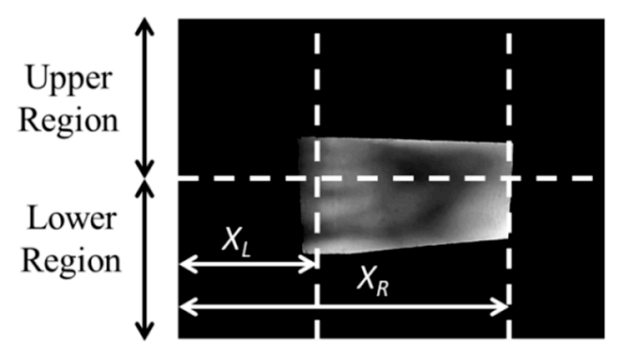

(c)

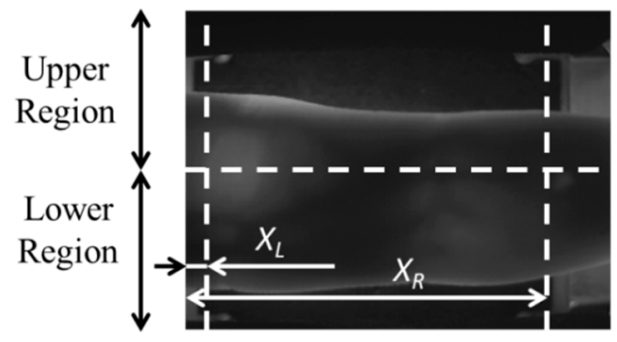

(e)

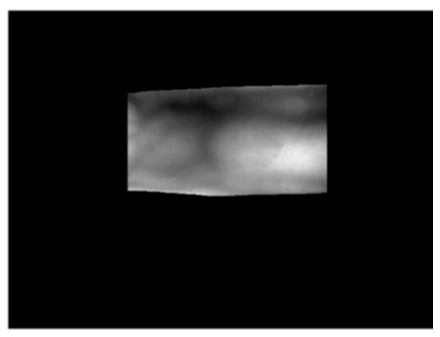

(b)

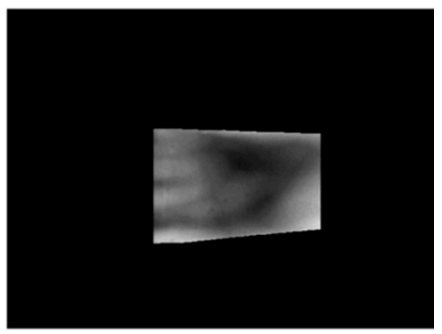

(d)

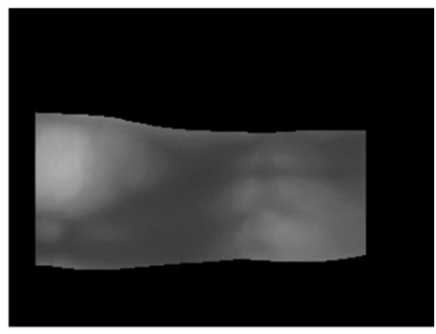

(f)

Figure 2. Examples of input finger-vein images and finger-region detection results obtained with images from the three databases: Original images from the (a) good-quality; (c) mid-quality; and (e) open databases with their corresponding finger-region detection results shown in $(\mathbf{b}, \mathbf{d}, \mathbf{f})$, respectively.

The 1st (Figure 2a) and 2nd database (Figure 2c) are collected by our lab-made devices (see Section 3). In our devices, each person puts his or her finger on the hole of the upper-part of device, and the size of the hole in the device for capturing the finger-vein image is fixed and limited in order to remove the effect by the environmental light into the captured image. Therefore, the part of the finger area can be acquired in the image, and the positions of left and right finger boundaries are restricted and same in all the captured images as shown in Figure 2a,c. Therefore, in order to enhance the processing speed of 
segmenting the finger area from the image, we use the pre-determined $X_{L}$ and $X_{R}$ values as the horizontal (X) position of the left and right boundary of the finger area, respectively.

In case of the 3rd database (Figure 2e), although the whole finger area can be acquired in the image, the left and right-most areas of finger are so dark (caused by the insufficient illumination of NIR light) that these areas are difficult to be used for finger-vein recognition. Therefore, we use the part of finger area by removing these left and right-most areas, based on pre-determined $X_{L}$ and $X_{R}$ values. The positions of the left and right boundaries can be automatically segmented with the 3rd database, but these positions can be different from each other among images, according to the performance of the segmentation algorithm of the finger area. The main goal of our research is not focused on the segmentation algorithm but on comparing the accuracies of recognition by assuming that images from the same person, the same hand, and the same finger types form the same classes. In addition, another goal is to compare the accuracies of recognition according to the number of finger-vein images combined by score-level fusion for recognition, and the number of images for enrollment. Therefore, we use the part of finger area by removing these left and right-most areas, based on pre-determined $X_{L}$ and $X_{R}$ values.

Then, two masks of $4 \times 20$ pixels, which are shown in Figure 3, were used to detect the upper and lower boundaries of the finger region. Because the gray level of the background region is lower than that of the finger region, as shown in Figure 2, the value that was calculated by using the masks in Figure 3 is maximized at the position of the finger boundary. Examples of the finger region detection results are given in Figure 2b,d,f.

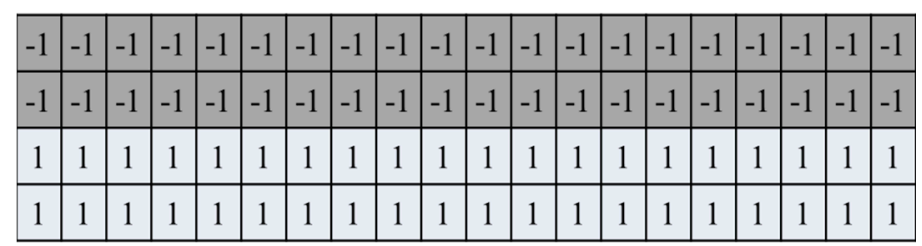

(a)

\begin{tabular}{|c|c|c|c|c|c|c|c|c|c|c|c|c|c|c|c|c|c|c|c|}
\hline 1 & 1 & 1 & 1 & 1 & 1 & 1 & 1 & 1 & 1 & 1 & 1 & 1 & 1 & 1 & 1 & 1 & 1 & 1 & 1 \\
\hline 1 & 1 & 1 & 1 & 1 & 1 & 1 & 1 & 1 & 1 & 1 & 1 & 1 & 1 & 1 & 1 & 1 & 1 & 1 & 1 \\
\hline-1 & -1 & -1 & -1 & -1 & -1 & -1 & -1 & -1 & -1 & -1 & -1 & -1 & -1 & -1 & -1 & -1 & -1 & -1 & -1 \\
\hline-1 & -1 & -1 & -1 & -1 & -1 & -1 & -1 & -1 & -1 & -1 & -1 & -1 & -1 & -1 & -1 & -1 & -1 & -1 & -1 \\
\hline
\end{tabular}

(b)

Figure 3. Masks for detecting finger-region boundaries in the vertical direction: Masks for detecting (a) the upper boundary; and (b) the lower boundary of the finger region.

Based on the detected finger boundaries, the finger-vein image is normalized to the size of $150 \times 60$ pixels by using a linear stretching method, and it is then sub-sampled to produce a $50 \times 20$ pixel image to enhance the processing speed [7]. This is done by averaging the gray values in each $3 \times 3$ pixel block of the $150 \times 60$ pixel image. Figure 4 shows examples of the normalization results of the images in Figure 2 . 


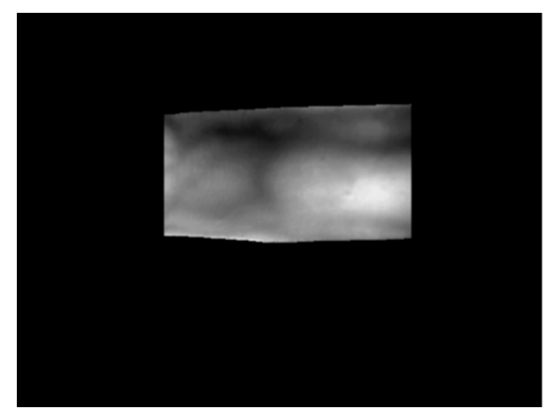

(a)

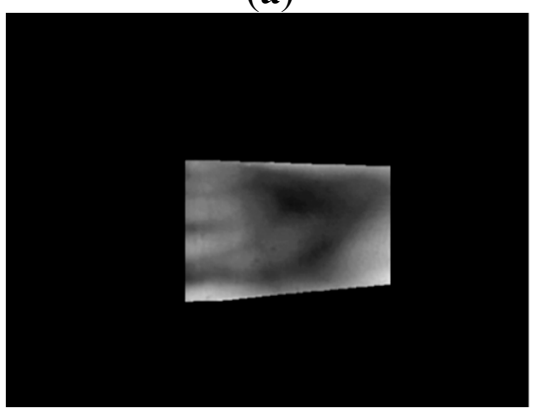

(d)

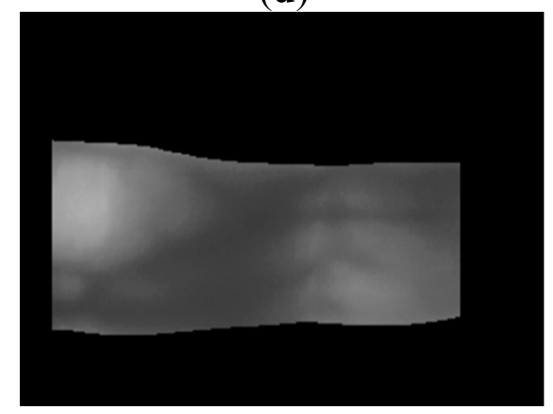

(g)

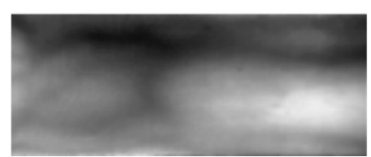

(b)

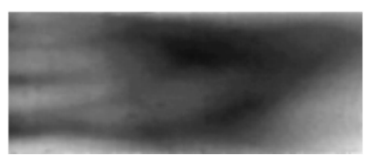

(e)

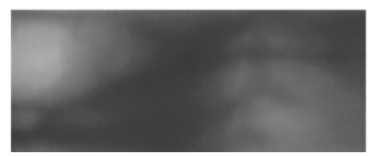

(h)

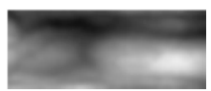

(c)

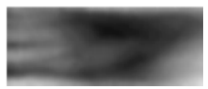

(f)

Figure 4. Linear stretching and sub-sampled results of finger-vein images from the three databases: Detected finger-region image from the (a) good-quality; (d) mid-quality; and (g) open databases, with their corresponding $150 \times 60$ pixel stretched images shown in $(\mathbf{b}, \mathbf{e}, \mathbf{h})$, respectively, and their corresponding $50 \times 20$ pixel sub-sampled images shown in $(\mathbf{c}, \mathbf{f}, \mathbf{i})$, respectively.

\subsection{Four-Directional Gabor Filtering}

Gabor filtering has been popularly used in finger-vein recognition for enhancing the image quality [6-8]. In this research, we apply a four-directional Gabor filter to the $50 \times 20$ pixel sub-sampled image prior to extracting the finger-vein code to enhance the distinctiveness of the vein image. Gabor filtering of the sub-sampled image could also be helpful to reduce the processing time compared to that of the original finger-vein image [7,8]. A two-dimensional Gabor filter can be represented as follows [6-8]:

$$
G(x, y)=\frac{1}{2 \pi \sigma_{x} \sigma_{y}} \exp \left\{-\frac{1}{2}\left(\frac{x_{\theta}^{2}}{\sigma_{x}^{2}}+\frac{y_{\theta}^{2}}{\sigma_{y}^{2}}\right)\right\} \exp \left(j 2 \pi f_{0} x_{\theta}\right)
$$

with 


$$
\left[\begin{array}{l}
x_{\theta} \\
y_{\theta}
\end{array}\right]=\left[\begin{array}{cc}
\cos \theta & \sin \theta \\
-\sin \theta & \cos \theta
\end{array}\right]\left[\begin{array}{l}
x \\
y
\end{array}\right]
$$

where $j=\sqrt{-1}, \theta$ is the direction, and $f_{0}$ is the central frequency of the Gabor kernel. The two coordinates $(x, y)$ are rotated to $x_{\theta}$ and $y_{\theta}$, respectively, and on each coordinate, the spatial envelopes of the Gaussian function are represented by $\sigma_{x}$ and $\sigma_{y}$, respectively. By eliminating the imaginary part of the Gabor filter, the real part, namely the even-symmetric Gabor filter, is used in this research because of the effectiveness with which it processes time. An even-symmetric Gabor filter is represented as Equation (2) as follows [6-8]:

$$
G_{k}^{E}(x, y)=\frac{1}{2 \pi \sigma_{x} \sigma_{y}} \exp \left\{-\frac{1}{2}\left(\frac{x_{\theta_{k}}^{2}}{\sigma_{x}^{2}}+\frac{y_{\theta_{k}}^{2}}{\sigma_{y}^{2}}\right)\right\} \cos \left(2 \pi f_{k} x_{\theta_{k}}\right)
$$

with

$$
\theta_{k}=k \pi / 4 ; k=1,2,3,4
$$

where $k$ is the index of the directional channel, and $\theta_{k}$ and $f_{k}$ represent the orientation and spatial frequency of the $k$ th channel, respectively. Based on previous research [6], the optimal parameters of $f_{k}$, $\sigma_{x}$, and $\sigma_{y}$, are determined to be $0.2,2.38$, and 2.38 , respectively, for the four channels in the $0^{\circ}, 45^{\circ}$, $90^{\circ}$, and $135^{\circ}$ directions of the Gabor filter applied to the sub-sampled image of $50 \times 20$ pixels. A convolution operation is applied to an input finger-vein image with the Gabor filter of the four channels to obtain the filtered image in the form of four separated convolution result images. These images are then combined by selecting, at each pixel position, the pixel with the lowest gray-level value among the four pixels of the four result images to be the final result of Gabor filtering, because, generally, the vein line is darker than the skin region [7]. Figure 5 provides example results of four-directional Gabor filtering on the sub-sampled images in Figure 4c,f,i.

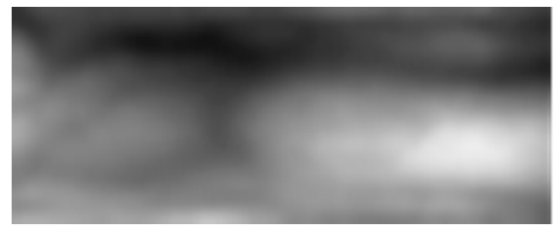

(a)

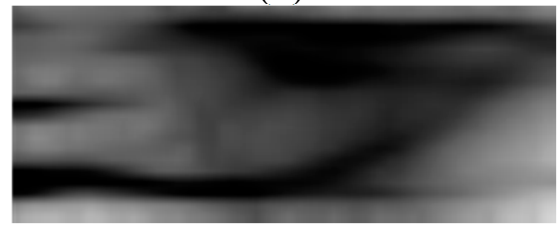

(d)

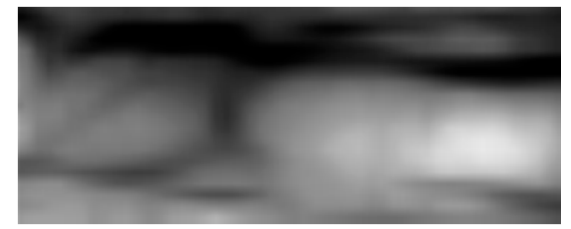

(b)

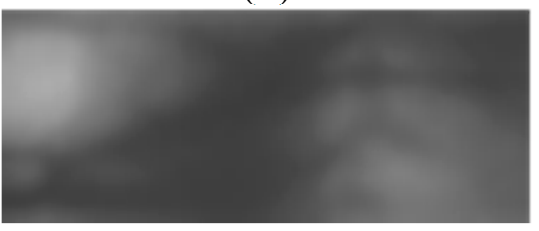

(e)

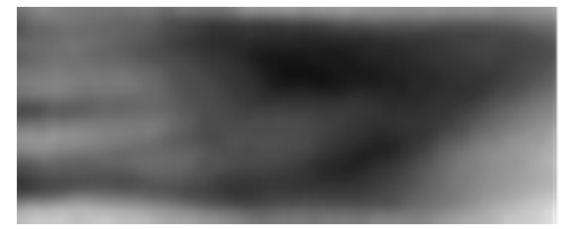

(c)

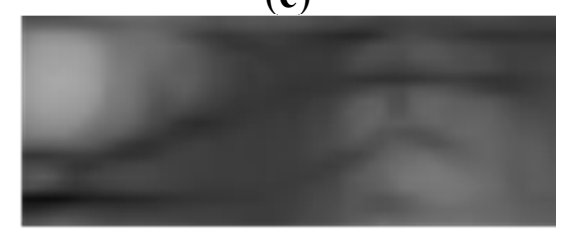

(f)

Figure 5. Gabor filtering results of the $50 \times 20$ pixel sub-sampled images from the three databases: Sub-sampled image from the (a) good-quality; (c) mid-quality; and (e) open databases with their respective Gabor filtered images shown in (b,d,f). 


\subsection{Finger-Vein Code Extraction Using LBP and Matching}

The binary codes are extracted from the quality enhanced finger-vein image by using the LBP method, which was selected based on its high performance [7]. This method encodes the difference between the gray level of each central pixel $\left(I_{C}\right)$ and that of its neighboring pixels $\left(I_{N}\right)$ to the binary values of 0 or 1 , as described by Equation (3) and illustrated in Figure 6. For each pixel position in a $50 \times 20$ pixel image, an 8-bit code string is extracted. Consequently, for each finger-vein image, a 6912-bit binary code ( 8 bits $\times 48$ columns $\times 18$ rows) is generated by the LBP operator.

$$
\begin{aligned}
& \operatorname{LBP}\left(x_{C}, y_{C}\right)=\sum_{k=0}^{7} S\left(I_{N}-I_{C}\right) \cdot 2^{k} \\
& S(t)= \begin{cases}1 & \text { if } \quad i \geq 0 \\
0 & \text { otherwise }\end{cases}
\end{aligned}
$$
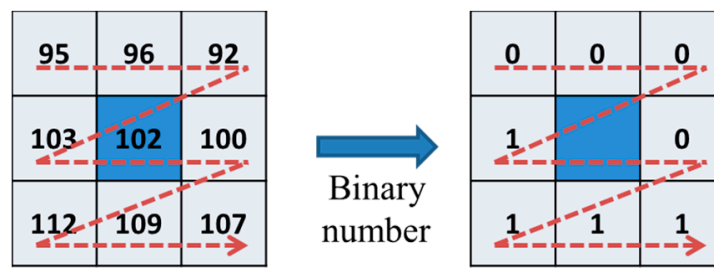

$\longrightarrow \begin{aligned} & \text { Binary code: } 11101000 \\ & \text { Decimal: } 232\end{aligned}$

Figure 6. LBP operator.

The matching distance is calculated by using the HD between the enrolled and input LBP binary codes. In this research, we used the normalized version of the Hamming distance on all of the 6912 bits of each finger-vein image as the following Equation (4) [7]:

$$
H D=\frac{V C E \oplus V C I}{N}
$$

where $V C E$ and $V C I$ are the binary codes extracted from the enrolled and input images, respectively, $\oplus$ is the Boolean exclusive OR (XOR) operator, and $N$ is the total number of bits (6912).

\section{Experimental Results}

\subsection{Proposed Finger-Vein Capturing Device}

Figure 7 depicts our finger-vein capturing device. This device consists of six NIR light-emitting diodes (LEDs) operating at a wavelength of $850 \mathrm{~nm}$ and a webcam (Logitech Webcam C600) [26]. Alignment of the input finger-vein image in the capturing process was achieved by attaching two bars to the device to guide the positioning of the fingertip and the side of the finger. This was done to ensure a high similarity between images acquired from the same finger of an individual and thus, increase the matching accuracy. By adding the guiding bars, our device is able to acquire finger-vein images for each person non-intrusively. This enabled us to create a good-quality finger-vein database with enhanced alignment of the finger position, and to use the database for the following experiments. 


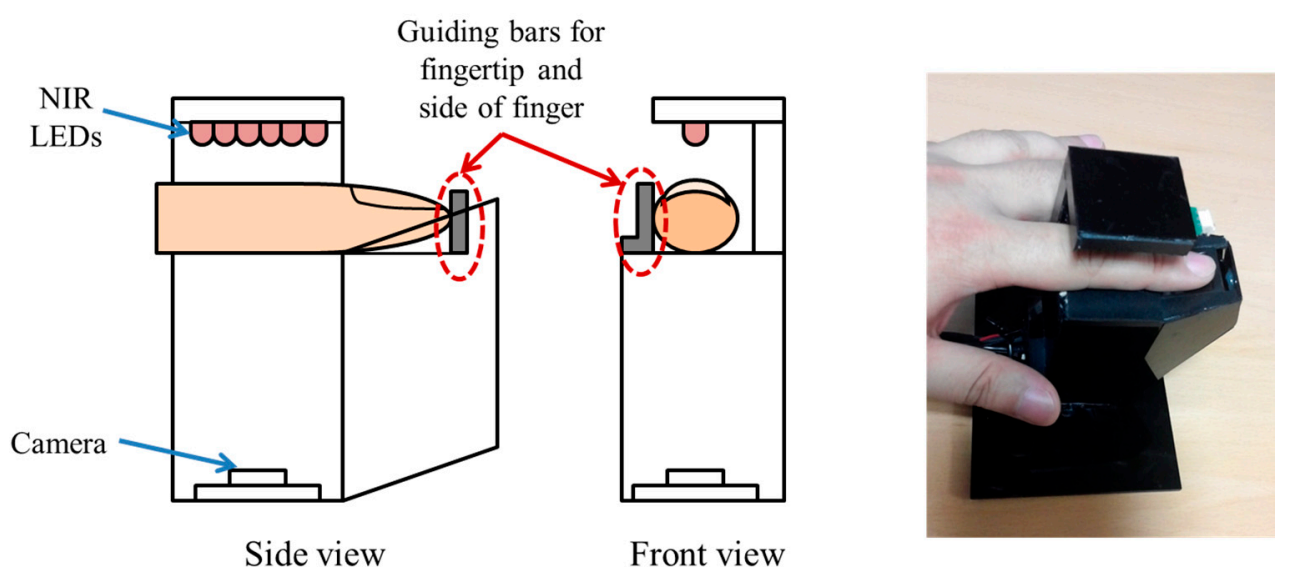

Figure 7. Proposed finger-vein capturing device used to build a good-quality database.

\subsection{Performance Evaluation on Three Databases}

For this research, we used three different finger-vein databases to evaluate the factors that affect the matching accuracy. The first database was created by collecting finger-vein data from 20 people using the device proposed in Section 3.1. [27]. For each person, the vein images of the index, middle, and ring fingers on both the left and right hands were captured 10 times with an image resolution of $640 \times 480$ pixels. The total number of images in our database was 1200 (20 people $\times 2$ hands $\times 3$ fingers $\times 10$ images) Because the finger alignment and image quality of the images in this database were strictly assured, it was considered a good-quality database.

In addition, we used two other databases, the first of which was constructed by selecting the vein images of six fingers among the images of 10 fingers in the database I (which were collected by the finger-vein capturing device without the guiding bar in previous research [7]). The device, which was used for collecting the database I [7], is shown in Figure 8. Because the guiding bar was absent, the misalignment among trial images of each finger in this database is relatively high; therefore, this was considered mid-quality database. In detail, each people puts his or her finger on the hole of the upper-part of device, and the size of the hole in the device for capturing the finger-vein image is fixed and limited in order to remove the effect by the environmental light into the captured image. Therefore, the part of finger area can be acquired in the image as shown in Figure 9a,b. Consequently, it is often the case that some part of the finger area (which is seen in the enrolled image) is not seen in the recognized image, which cannot be compensated by preprocessing step and can reduce the accuracy of recognition. In order to solve this problem, we propose a new device including two guiding bars for fingertip and side of finger as shown in Figure 7, which can make the consistent finger area be acquired by our device with reduced misalignment. However, no guiding bar is used in the other device of Figure 8 , which is used for collecting the 2 nd database. Therefore, we call the 1 st and 2 nd databases collected by the devices of Figures 7 and 8 as good-quality and mid-quality databases, respectively.

The number of images in the mid-quality database is 1980 ( 33 people $\times 2$ hands $\times 3$ fingers $\times 10$ trials), and each image has the same size as that of the images in the good-quality database, i.e., $640 \times 480$ pixels [27]. 


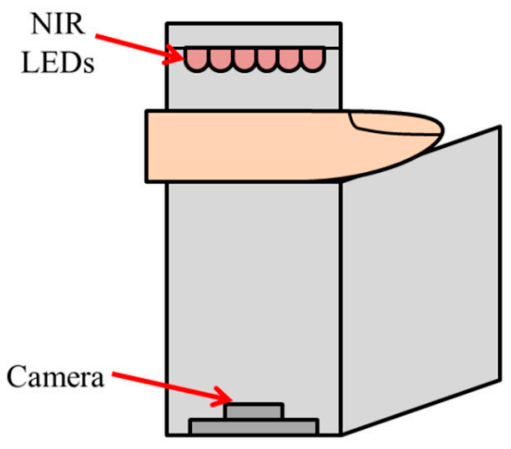

Side view

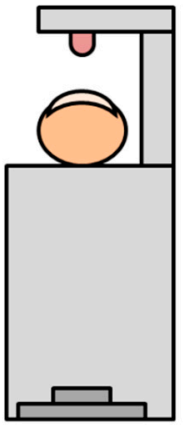

Front view

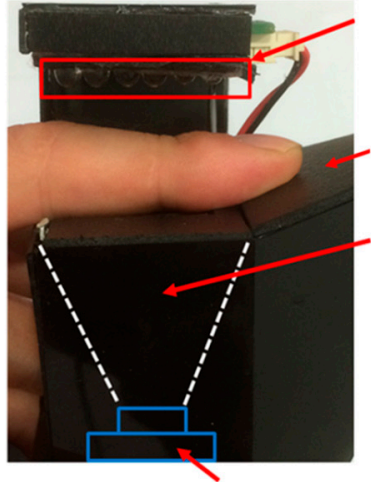

Web camera
NIR illuminator of 6 LEDs

Board for touching the part between fingertip and the $1^{\text {st }}$ knuckle of finger

Capturing device for finger-vein image

Figure 8. Device for capturing finger-vein images for the second (mid-quality) database.

The last database used in this study is an open finger-vein database (SDUMLA-HMT Finger-vein database) [28], which comprises 3816 images, with a size of $320 \times 240$ pixels, from 106 people, including six fingers from each person and six trials for each finger. Example images of different trials of one individual (same finger) from each database are given in Figure 9. It can be seen in Figure 9 that the degree of misalignment among the trials of each finger from the mid-quality and open databases is larger than that from the good-quality database.
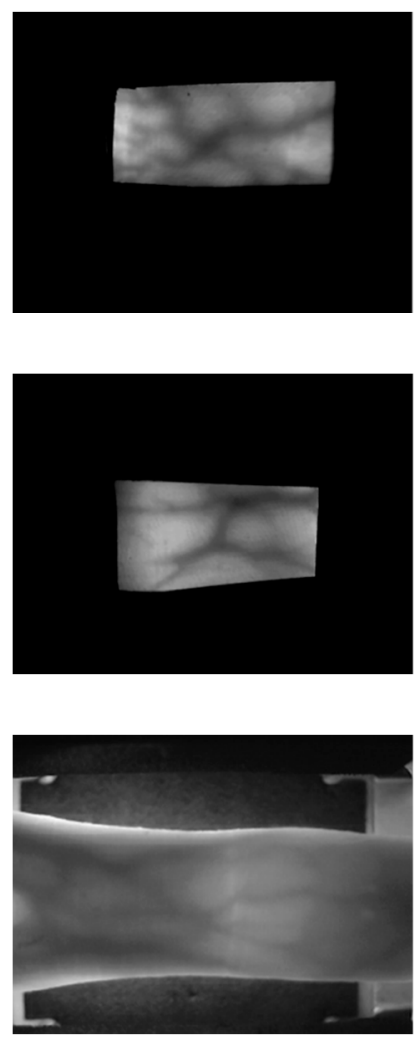

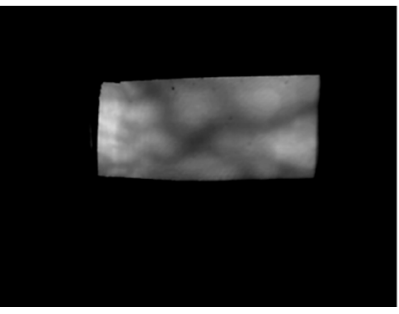

(a)

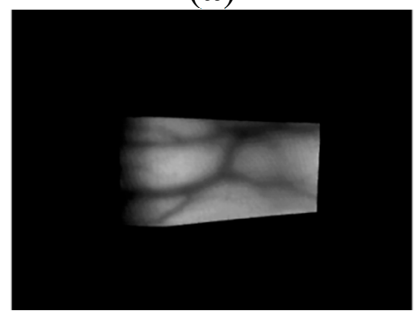

(b)

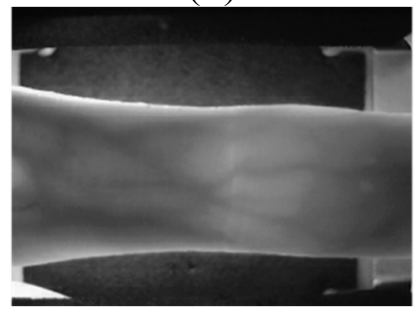

(c)
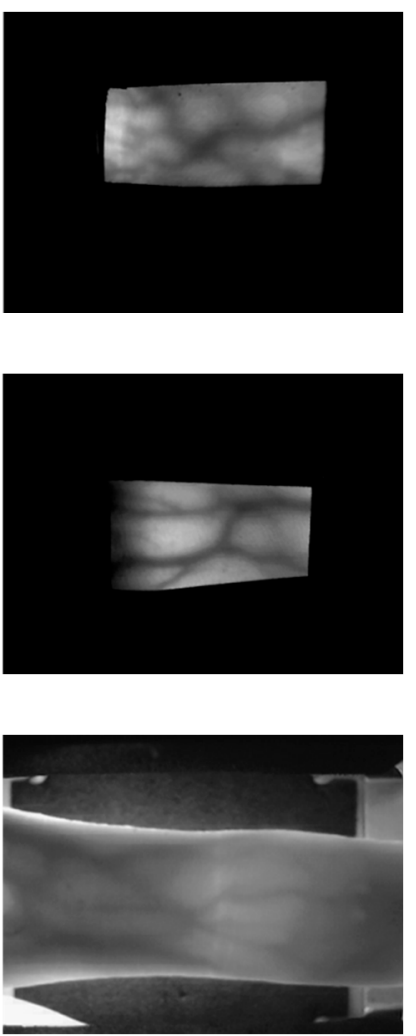

Figure 9. Input images of different trials from the same finger of one individual from each database: (a) good-quality; (b) mid-quality; and (c) open database. 
The accuracies of the finger-vein recognition method were evaluated by performing authentic and imposter matching tests. In our experiments, the images in each finger-vein database could be classified in various ways to allow the discrimination factors to be evaluated. In each experiment, authentic matching tests were used to calculate the pairwise matching distances between the images selected from the same class, whereas for the imposter matching tests, the distances between the pairs of images from different classes were calculated. Assuming that for a particular database, we classify finger-vein images into $M$ classes and each class has $N$ images, then the number of authentic and imposter matching tests denoted by $A$ and $I$ are determined by the following Equations (5) and (6), respectively.

$$
\begin{gathered}
A={ }_{N} C_{2} \times M \\
I=N \times{ }_{M} C_{2} \times N
\end{gathered}
$$

where ${ }_{N} C_{2}=N(N-1) / 2$ is the number of two-combinations from a set of $N$ elements.

By applying and adjusting the threshold on the matching Hamming distance, we calculated the false acceptance rates (FARs), false rejection rates (FRRs), and the EER. FAR refers to the error rates of imposter matching cases, which are misclassified into authentic classes, whereas FRR refers to the error rates of misclassified authentic testing cases into imposter classes. EER is the error rate when the difference between FAR and FRR is minimized. In addition, we measured the d-prime $\left(d^{\prime}\right)$ value, which represents the classifying ability between authentic and imposter matching distributions as the following Equation (7) [3].

$$
d^{\prime}=\frac{\mu_{A}-\mu_{I}}{\sqrt{\frac{\sigma_{A}^{2}+\sigma_{I}^{2}}{2}}}
$$

where $\mu_{A}$ and $\mu_{I}$ represent the mean values of the authentic and imposter matching distributions, respectively, and $\sigma_{A}$ and $\sigma_{I}$ denote the standard deviations of authentic and imposter matching distributions, respectively. A higher d-prime value indicates a larger separation between the authentic and imposter matching distributions, which corresponds to a lower error of recognition, in case that the distributions of authentic and imposter matching scores are similar to Gaussian shape, respectively.

We conducted the following experiments to evaluate the various factors that affect the results of the finger-vein recognition system.

First, we considered each finger of each person to form a different class. This method is used by conventional finger-vein recognition systems to evaluate the recognition accuracy [7-9,11,16-23,25]. Consequently, for the good-quality, mid-quality, and open databases, the number of classes were 120 (20 people $\times 6$ fingers), 198 (33 people $\times 6$ fingers), and 636 (106 people $\times 6$ fingers), respectively. As this class definition method includes the dissimilarity information of fingers, hands, and people in the finger-vein database, we considered this as the 1st experiment (classified by fingers, hands, and people).

In the 2nd experiment, we classified the finger-vein images based on people (classified by people), by assuming that the images of all the fingers on both hands from the same person formed the same class. As a result, in the 2 nd experiment, the number of classes in each database equaled the number of users, which was 20,33, and 106 for the good-quality, mid-quality, and open databases, respectively. 
In the 3rd experiment, we assumed that the finger-vein images of all the fingers on the left hands of all the people belong to the same class, and those on the right hands of all the people form another class. Thus, there were two classes based on different hand sides in this experiment (classified by hands).

In the 4th experiment, we evaluated the dissimilarities of finger types by assuming that the images from the index fingers, middle fingers, and ring fingers on both hands of all the people belong to three different classes. This assumption is referred to as (classified by fingers). The organization of these experiments is summarized in Figure 10. The numbers of authentic and imposter matching tests in the experiments on the three databases are determined by Equations (5) and (6), and are shown in Table 2.

Table 2. Number of matching tests (authentic and imposter) for the experiments on the three finger-vein databases ( $M$ is the number of classes in each experiment and $N$ is the number of images belonging to one class. Authentic and Imposter refer to the numbers of authentic and imposter matching tests, respectively).

\begin{tabular}{cccccc}
\hline \multirow{2}{*}{ Experiments Databases } & 1st Experiment & 2nd Experiment & 3rd Experiment & 4th Experiment \\
\cline { 3 - 6 } & & $\begin{array}{c}\text { Classified by Fingers, } \\
\text { Hands and People }\end{array}$ & $\begin{array}{c}\text { Classified by } \\
\text { People }\end{array}$ & $\begin{array}{c}\text { Classified by } \\
\text { Hands }\end{array}$ & $\begin{array}{c}\text { Classified by } \\
\text { Fingers }\end{array}$ \\
\hline \multirow{2}{*}{ Good-quality } & $N / M$ & $10 / 120$ & $60 / 20$ & $600 / 2$ & $400 / 3$ \\
Database & Authentic & 5400 & 35,400 & 359,400 & 239,400 \\
& Imposter & 714,000 & 684,000 & 360,000 & 480,000 \\
\hline \multirow{2}{*}{ Mid-quality } & $N / M$ & $10 / 198$ & $60 / 33$ & $990 / 2$ & $660 / 3$ \\
Database & Authentic & 8910 & 58,410 & 979,110 & 652,410 \\
& Imposter & $1,950,300$ & $1,900,800$ & 980,100 & $1,306,800$ \\
\hline \multirow{2}{*}{ Open Database } & Authentic & $6 / 636$ & $36 / 106$ & $1908 / 2$ & $1272 / 3$ \\
& Imposter & $7,269,480$ & 66,780 & $3,638,556$ & $2,425,068$ \\
\hline
\end{tabular}

Table 3 shows the comparative results of the four experiments defined in Table 2 and Figure 10 for the three databases. In the 1st experiment, in which finger-vein images were classified by fingers, hands, and people, the lowest EER $(0.474 \%)$ was obtained for the good-quality database. This is due to the fact that this database was captured by the proposed capturing device, which uses a guiding bar to reduce the misalignment among input finger-vein images. In the case of the open database, the authors did not apply any guiding bar for alignment in the image-capturing device [29]. As a result, the EER obtained from this database was the highest $(8.096 \%)$ because of the misalignment of captured fingers. The results of the first experiment also indicate that the matching accuracies from images in the good-quality database were the highest, followed by those in the mid-quality database, whereas the worst matching accuracies were obtained for the open database, in terms of EERs $(0.474 \%, 2.393 \%$, and $8.096 \%$, respectively). These results correspond to the level of misalignment in each finger-vein database. The resulting plots of the ROC curves and matching distance distributions obtained from the experiments classified by fingers, hands, and people for the three databases are shown in Figures 11 and 12. 


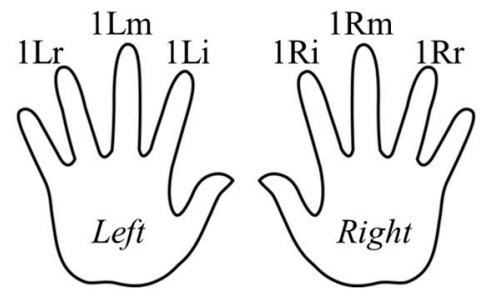

Person 1

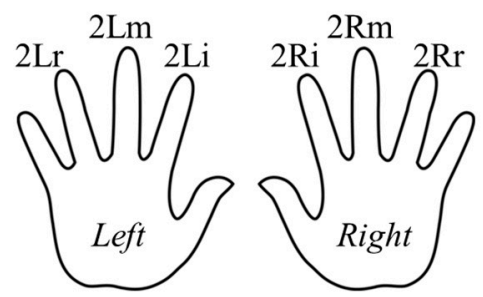

Person 2

$<$ Finger descriptions $>$

Lr - Left Ring, Lm - Left Middle, Li - Left Index;

$\mathrm{Rr}$ - Right Ring, Rm - Right Middle, Ri - Right Index;

$1^{\text {st }}$ experiment (Classified by Fingers, Hands, and People):

Class $1=\{1 \mathrm{Lr}\}$

Class2 $=\{1 \mathrm{Lm}\}$

Class $3=\{1 \mathrm{Li}\}$;

$\ldots$

- $\quad 2^{\text {nd }}$ experiment (Classified by People):

Class1 (person1) $=\{1 \mathrm{Lr}, 1 \mathrm{Lm}, 1 \mathrm{Li}, 1 \mathrm{Ri}, 1 \mathrm{Rm}, 1 \mathrm{Rr}\}$;

Class 2 (person2) $=\{2 \mathrm{Lr}, 2 \mathrm{Lm}, 2 \mathrm{Li}, 2 \mathrm{Ri}, 2 \mathrm{Rm}, 2 \mathrm{Rr}\}$;

...

$3^{\text {rd }}$ experiment (Classified by Hands):

Class1 (left hand $)=\{1 \mathrm{Lr}, 1 \mathrm{Lm}, 1 \mathrm{Li}, 2 \mathrm{Lr}, 2 \mathrm{Lm}, 2 \mathrm{Li}, \ldots\}$;

Class 2 (right hand) $=\{1 \mathrm{Ri}, 1 \mathrm{Rm}, 1 \mathrm{Rr}, 2 \mathrm{Ri}, 2 \mathrm{Rm}, 2 \mathrm{Rr}, \ldots\}$;

- $4^{\text {th }}$ experiment (Classified by Fingers):

Class1 (index) $=\{1 \mathrm{Li}, 1 \mathrm{Ri}, 2 \mathrm{Li}, 2 \mathrm{Ri}, \ldots\}$;

Class2 $($ middle $)=\{1 \mathrm{Lm}, 1 \mathrm{Rm}, 2 \mathrm{Lm}, 2 \mathrm{Rm}, \ldots\}$;

Class3 (ring) $=\{1 \mathrm{Lr}, 1 \mathrm{Rr}, 2 \mathrm{Lr}, 2 \mathrm{Rr}, \ldots\}$;

Figure 10. Organization of experiments for finger-vein database.

Table 3. Comparative results of the four experiments for the three databases.

\begin{tabular}{|c|c|c|c|c|c|c|c|}
\hline & \multirow{2}{*}{ Experiments } & \multicolumn{2}{|c|}{ Good-Quality Database } & \multicolumn{2}{|c|}{ Mid-Quality Database } & \multicolumn{2}{|c|}{ Open Database } \\
\hline & & $\operatorname{EER}(\%)$ & d-Prime & $\operatorname{EER}(\%)$ & d-Prime & $\operatorname{EER}(\%)$ & d-Prime \\
\hline 1st Experiment & Classified by Fingers, Hands, and People & 0.474 & 5.805 & 2.393 & 4.022 & 8.096 & 2.727 \\
\hline 2nd Experiment & Classified by People & 40.223 & 0.695 & 39.280 & 0.759 & 36.095 & 0.791 \\
\hline 3rd Experiment & Classified by Hands & 48.427 & 0.136 & 49.320 & 0.072 & 49.137 & 0.039 \\
\hline 4th Experiment & Classified by Fingers & 45.434 & 0.277 & 45.506 & 0.267 & 47.299 & 0.147 \\
\hline
\end{tabular}

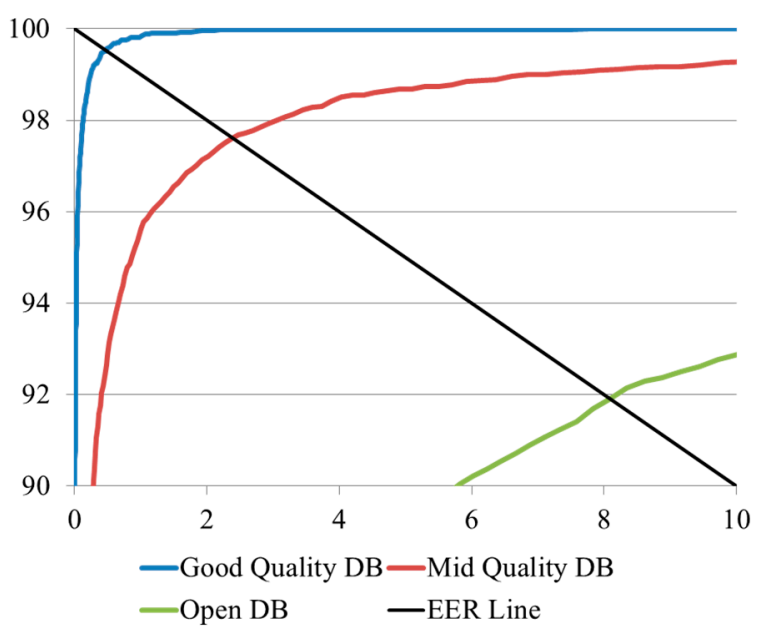

Figure 11. ROC curves of the 1st experiment on the three databases (DBs).

In the 2nd experiment, we classified the finger-vein images from the three databases based on people. In this way, the finger-vein images from the same person were considered as belonging to the same class; hence, the finger-vein images in different classes indicated the dissimilarities between different people. Likewise, the 3rd and 4th experiments on the three databases, considered images from the same hand 
side (i.e., either the left or the right hand), and images from the same finger type (i.e., the index, middle, or ring fingers) of all the people to be from the same classes, respectively. A comparison of the results of the three experiments (2nd, 3rd and 4th) on each database by considering the finger-vein dissimilarity between people, hands, and fingers, enabled us to evaluate the effect of each of these factors on the accuracy of the finger-vein recognition system.

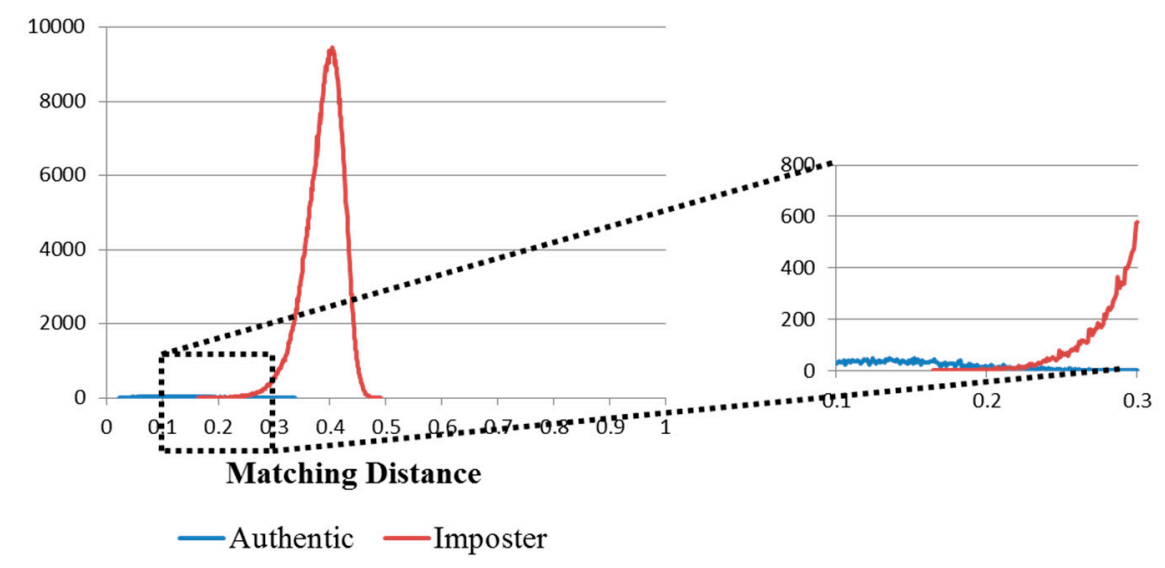

(a)

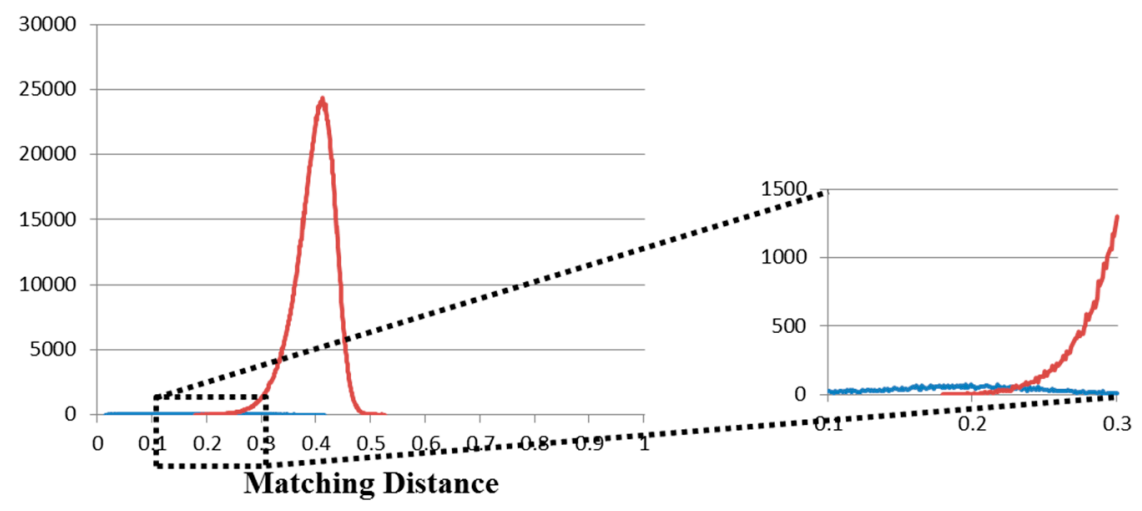

- Authentic Imposter

(b)

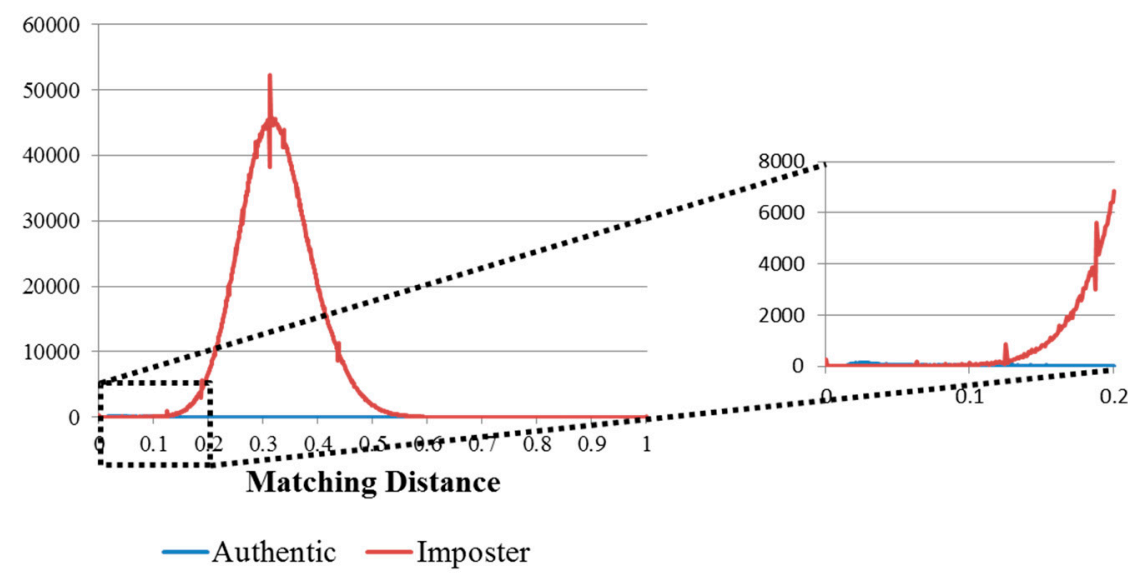

(c)

Figure 12. Matching distance distribution of authentic and imposter matching tests in the $1 \mathrm{st}$ experiment on the three databases: (a) good-quality; (b) mid-quality; and (c) open database. 


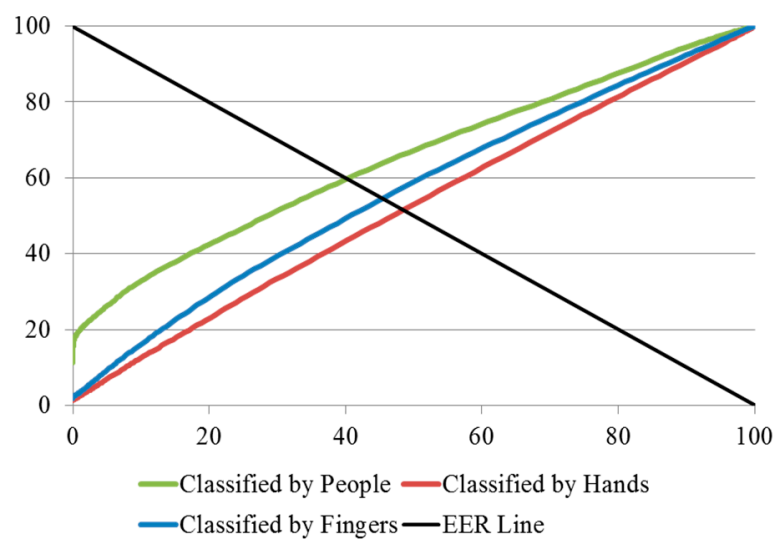

(a)

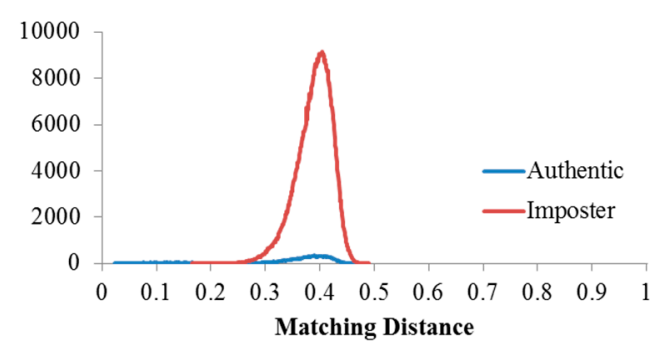

(b)

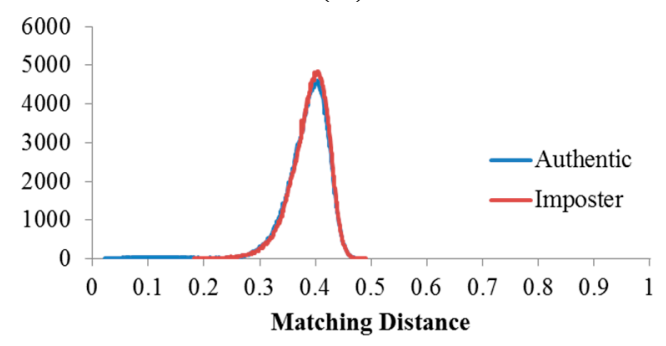

(c)

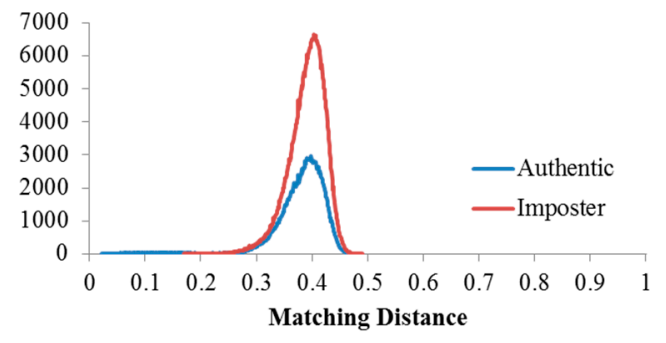

(d)

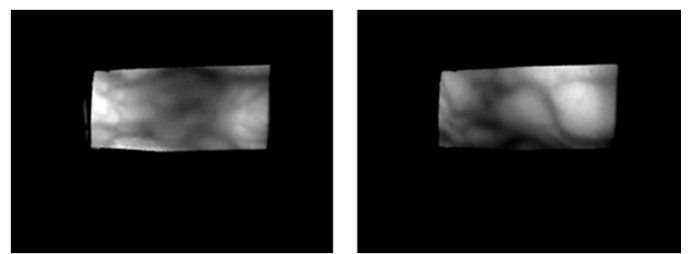

(e)
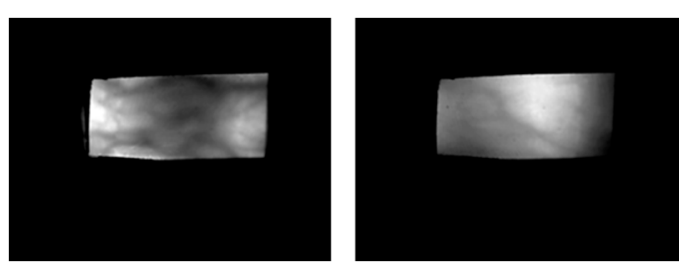

(f)
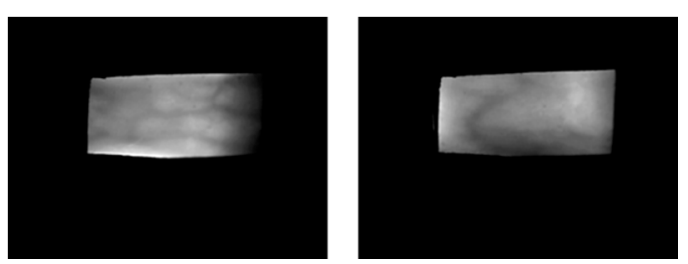

(g)

Figure 13. Results of the 2nd, 3rd and 4th experiments on the good-quality database: (a) ROC curves of the results of the three experiments; matching distribution of (b) the experiment classified by people (2nd experiment); (c) the experiment classified by hands (3rd experiment); and (d) the experiment classified by fingers (4th experiment), each shown with its corresponding false rejection error case: (e) images of the ring and index fingers on left hand of the same person; (f) images of the ring and index fingers on left hands of two different people; and (g) images of the middle fingers on left and right hands of two different people. 


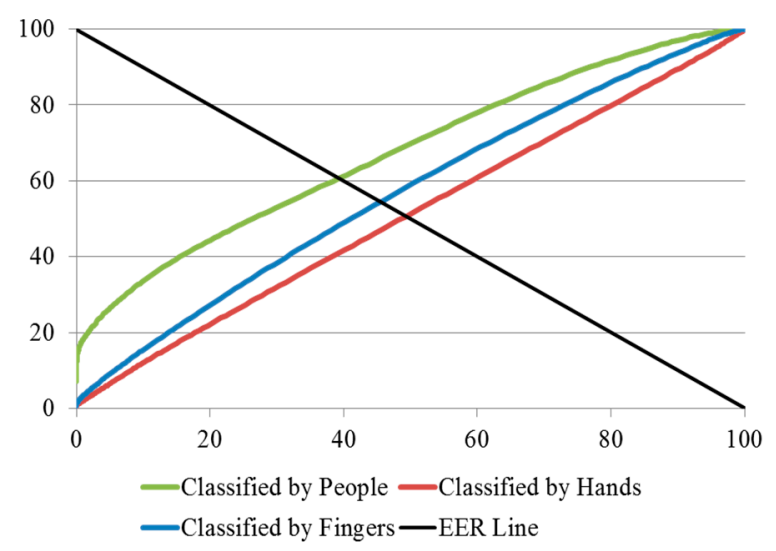

(a)

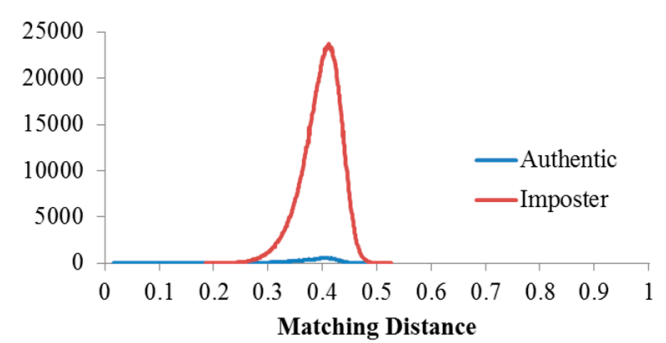

(b)

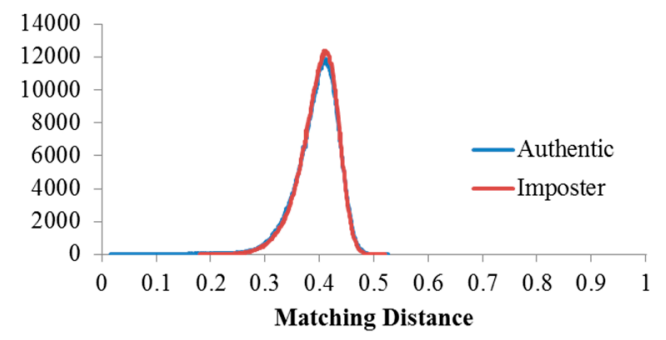

(d)

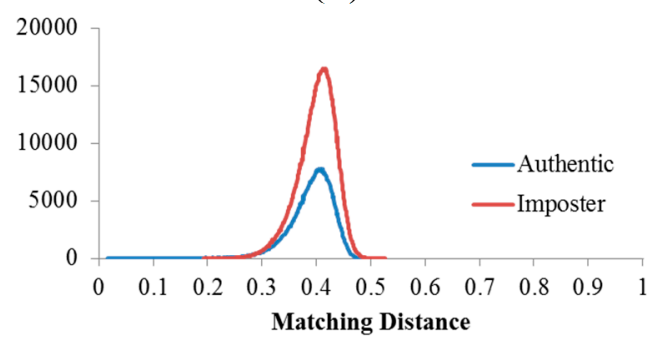

(f)
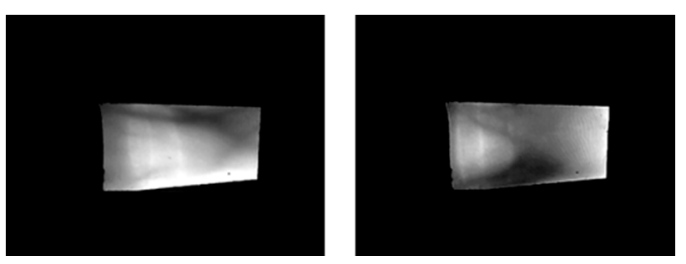

(c)
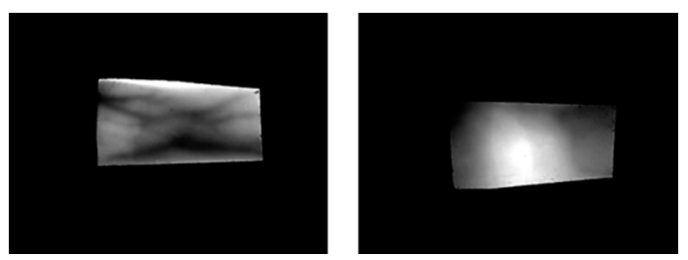

(e)
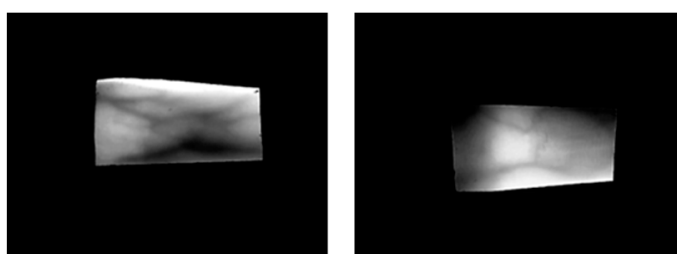

$(\mathbf{g})$

Figure 14. Results of the $2 \mathrm{nd}, 3 \mathrm{rd}$, and 4 th experiments on the mid-quality database: (a) ROC curves of the results of the three experiments; matching distribution of (b) the experiment classified by people (2nd experiment); (d) the experiment classified by hands (3rd experiment); and (f) the experiment classified by fingers (4th experiment), each shown with its corresponding false rejection error case: (c) images of the right ring and left middle fingers of the same person; (e) images of the ring and index fingers on right hands of two different people; and (g) images of the ring fingers on right hands of two different people. 


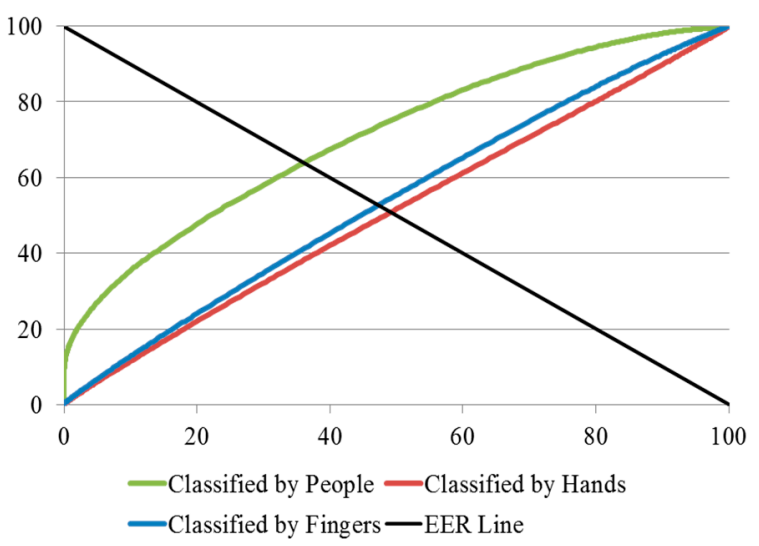

(a)

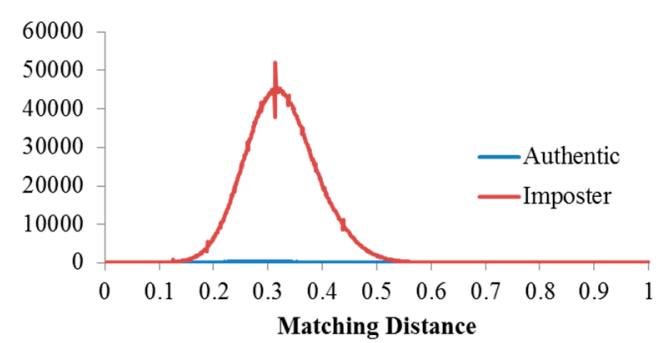

(b)

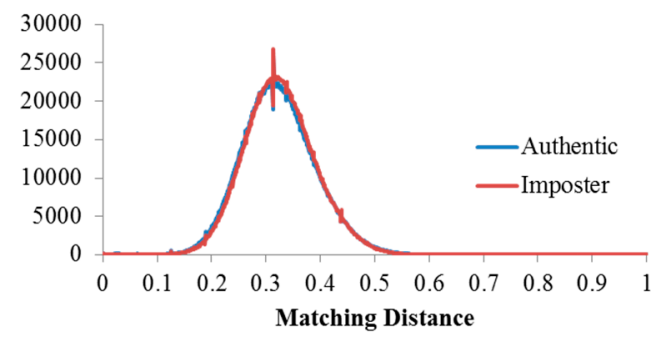

(d)

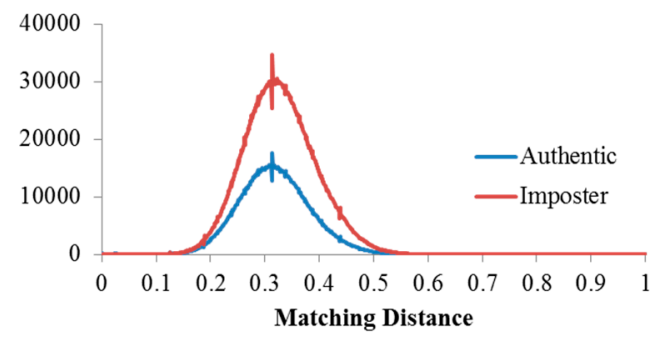

(f)

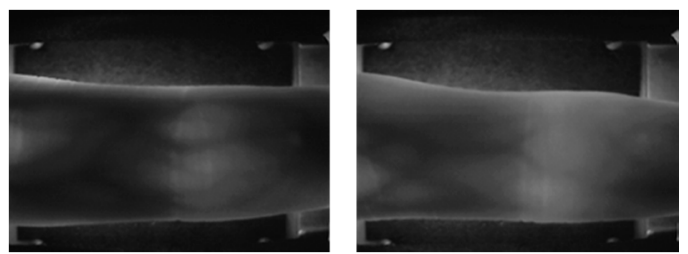

(c)
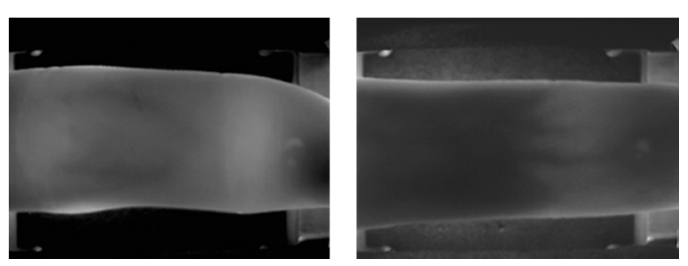

(e)
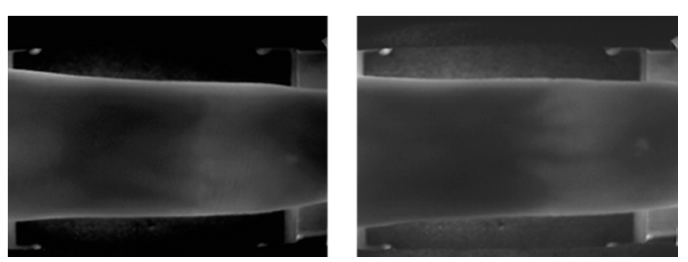

(g)

Figure 15. Results of the 2nd, 3rd, and 4th experiments on the open database: (a) ROC curves of the results of the three experiments; matching distribution of (b) the experiment classified by people (2nd experiment); (d) the experiment classified by hands ( $3 \mathrm{rd}$ experiment); and (f) the experiment classified by fingers (4th experiment); each shown with its corresponding false rejection error case: (c) images of the right middle and right ring fingers of the same person; (e) images of the index and middle fingers on left hands of two different people; and (g) images of the middle fingers on two hands of two different people.

Table 3 indicates that, when the three databases are compared, the lowest EERs (the highest d-prime value) were obtained from the experiment classified by people (the 2 nd experiment), the second 
lowest EERs (the second highest d-prime value) were obtained from the experiment classified by fingers (the 4th experiment), and that classified by hands (3rd experiment) produced the highest EERs (the lowest d-prime value). This sequence was consistent for all three of the databases.

Consequently, we are able to conclude that, finger-vein dissimilarity increases in the order people, fingers, and hands, respectively. In other words, the discrimination between finger-vein images from different people is larger than that between the different finger types (index, middle, and ring fingers) and that between hands from different sides (left or right hands).

The plots of the ROC curves and matching distributions of authentic and imposter tests obtained from the three experiments (the 2nd, 3rd, and 4th experiments) as well as the error cases for the good-quality, mid-quality, and open databases are shown in Figures 13-15, respectively. In the 2nd experiment (classified by people), the cases for which a false rejection was obtained were for different fingers from the same person. The false rejection cases of the 3rd experiment (classified by hands) were the matching pair of vein images of fingers from the same hand side, but belonging to different people or captured from different fingers. Similarly, the false rejections of the 4th experiment (classified by fingers) are cases in which images were captured from the same finger types (i.e., index, middle, or ring fingers) but belonged to different people or hand sides.

\subsection{Experimental Results Using Multiple Images for Enrollment}

In this experiment, we used a number of input finger-vein images for enrollment instead of using only one image as was done previously [7-9,11,16-23,25]. The method involving the enrollment of finger-vein data using the average of multiple finger-vein images is as follows. After the input images were captured for enrollment, they were processed and normalized by the methods described in Sections 2.2 and 2.3. From the image consisting of $50 \times 20$ pixels, obtained as a result of sub-sampling, we obtained the average image from which we extracted the LBP code which was then enrolled into the system. We applied this method by using either three or five enrollment finger-vein images to compare the matching accuracies with the conventional method, which only uses one image for enrollment. Examples of the average images generated from the $50 \times 20$ pixel images are shown in Figure 16. The experiments were conducted on the good-quality database as demonstrated in Figure 9a.
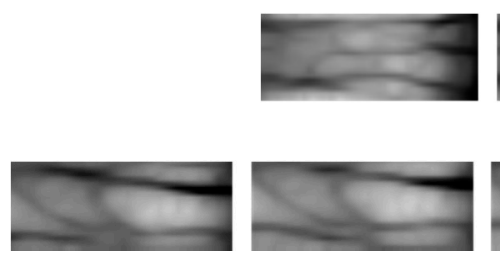
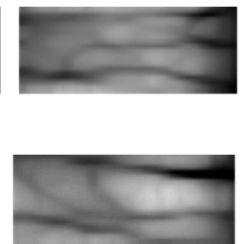

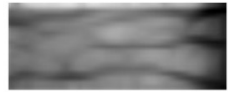

(a)

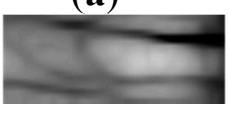

(b)
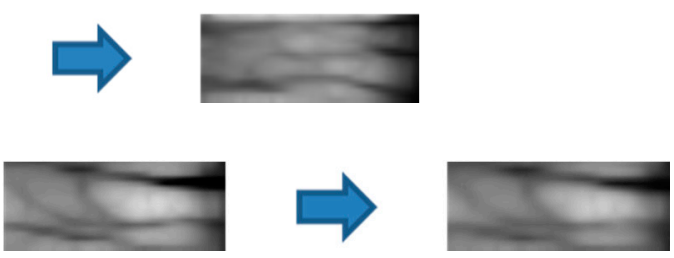

Figure 16. Normalized finger-vein images and their average images when: (a) three images; and (b) five images were used for enrollment.

When three images were used for enrollment, these were selected from the 10 images of each finger of the same user. Then, we extracted the finger-vein code from the average of these three images, and used the data extracted from the remaining seven images of the same finger to perform authentic matching tests. For the imposter matching tests, we used the images of the other fingers to perform 
matching with the average image generated for the enrolled finger. This experimental method is illustrated in Figure 17.

Assuming that the images from different fingers, hands, and people belong to different classes, the good-quality database contained 120 classes in total, as shown in Table 2 . When three images were used for enrollment, the number of authentic matching tests was $100,800\left({ }_{10} \mathrm{C}_{3} \times 7\right.$ (the number of remaining images in the same class) $\times 120$ (the number of classes)), whereas the number of imposter matching tests was $17,136,000\left({ }_{10} \mathrm{C}_{3} \times 10\right.$ (the number of images in other classes) $\times 119$ (the number of other classes) $\times 120$ (the number of classes from which images for enrollment were selected)).

When five images were used for enrollment, the number of authentic matches was 151,200 $\left({ }_{10} \mathrm{C}_{5} \times 5\right.$ (the number of remaining images in the same class) $\times 120$ (the number of classes)), whereas that of imposter matches was 35,985,600 $\left({ }_{10} \mathrm{C}_{5} \times 10\right.$ (the number of images in other classes) $\times 119$ (the number of other classes $) \times 120$ (the number of classes from which images for enrollment were selected))

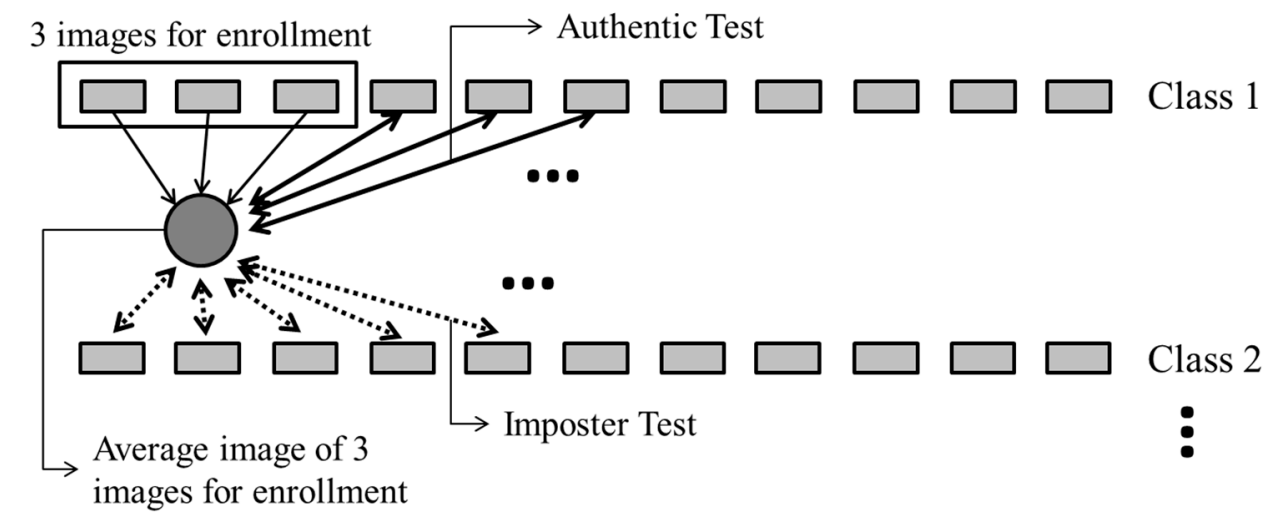

Figure 17. Experimental method when three images were used for finger-vein enrollment.

The experimental results of the methods in which three and five images were used for enrollment, are compared with those obtained by the conventional method (using one image for enrollment) in Table 4, where it can be seen that the matching accuracy was enhanced by increasing the number of enrollment images, in terms of low EER and high d-prime values. The ROC curves and the distribution plots of authentic and imposter tests corresponding to the results in Table 4 are shown in Figures 18 and 19, respectively, and can be explained as follows. In the finger-vein database, matching errors were mostly caused by misalignment at the time when the input finger-vein images were initially recorded, which subsequently resulted in translation errors in the normalized images of $50 \times 20$ pixels. The use of image averaging reduced the translation errors within the normalized images and increased the similarities between the enrolled and the matched finger-vein data. Table 5 shows examples of error cases resulting in false rejection when the enrolled images were compared with the test image in the same class, listed according to the number of images used for enrollment.

Table 4. Comparative results when multiple images were used for enrollment.

\begin{tabular}{lll}
\hline Number of Images for Enrollment & EER (\%) & d-Prime \\
\hline 1 & 0.474 & 5.805 \\
3 & 0.454 & 6.406 \\
5 & 0.362 & 6.633 \\
\hline
\end{tabular}




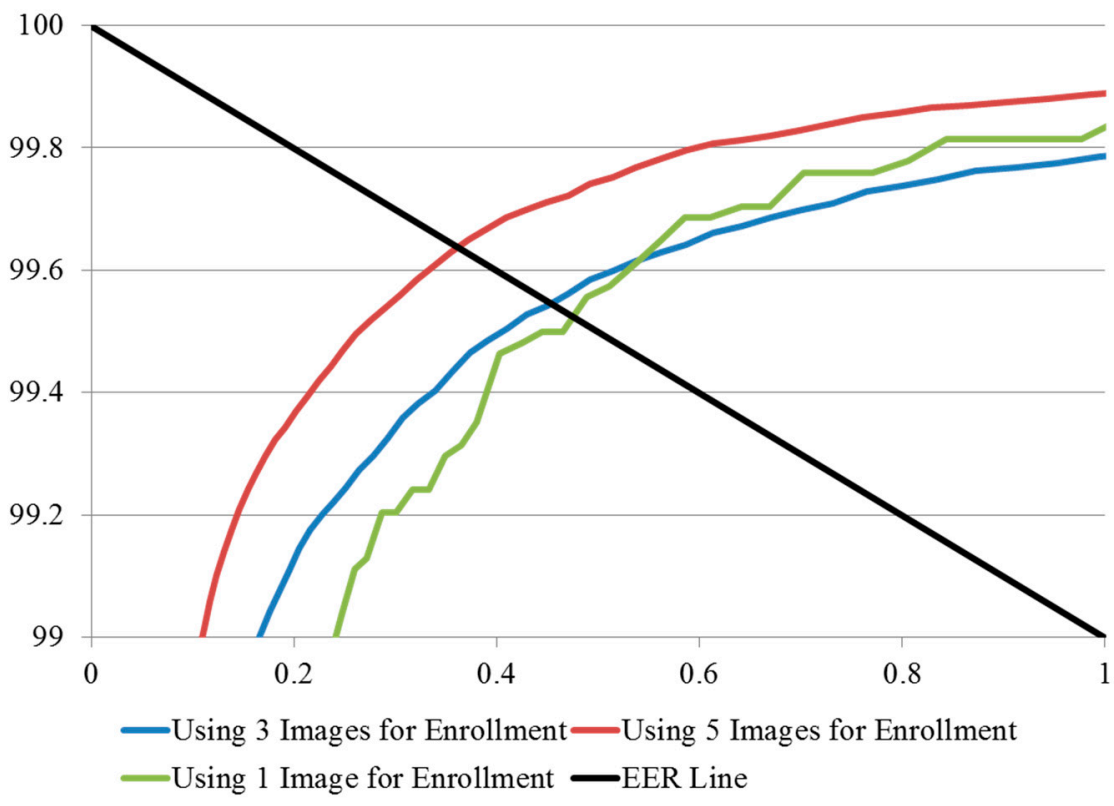

Figure 18. ROC curves of using multiple images for enrollment methods.

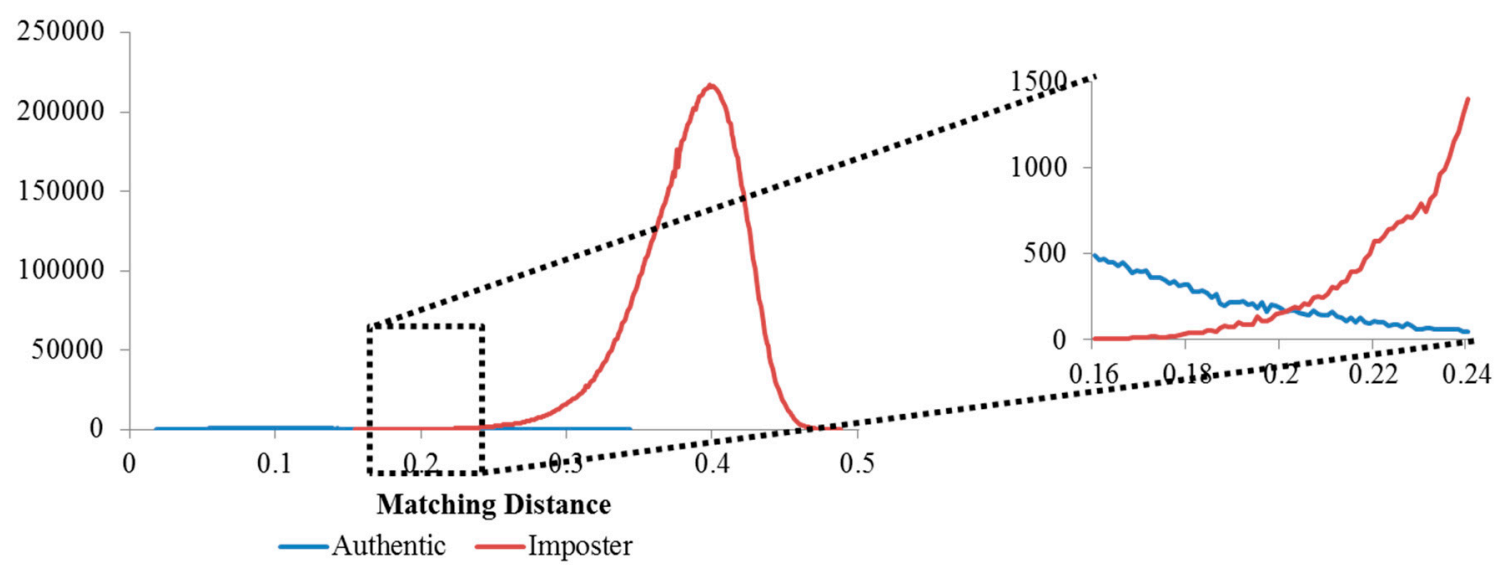

(a)

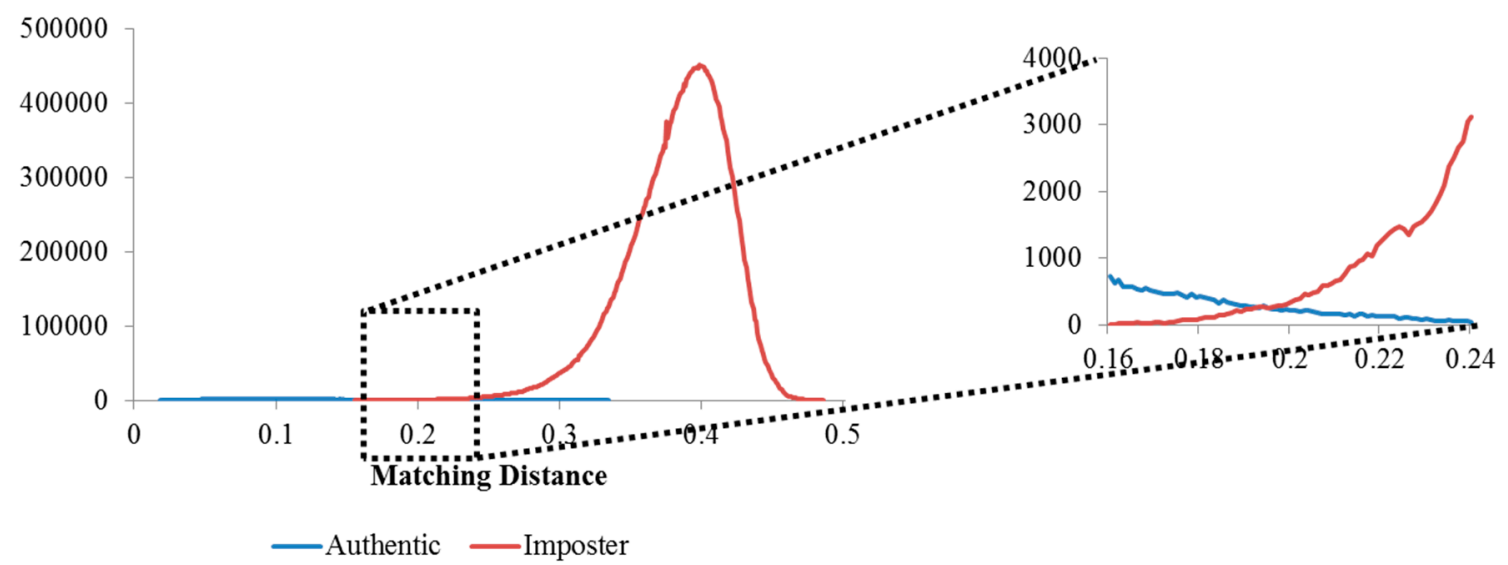

(b)

Figure 19. Matching distance distributions of authentic and imposter tests using (a) three images; and (b) five images for enrollment methods. 
Table 5. False rejection cases: Comparison of the detected input finger-vein images with the enrolled images.

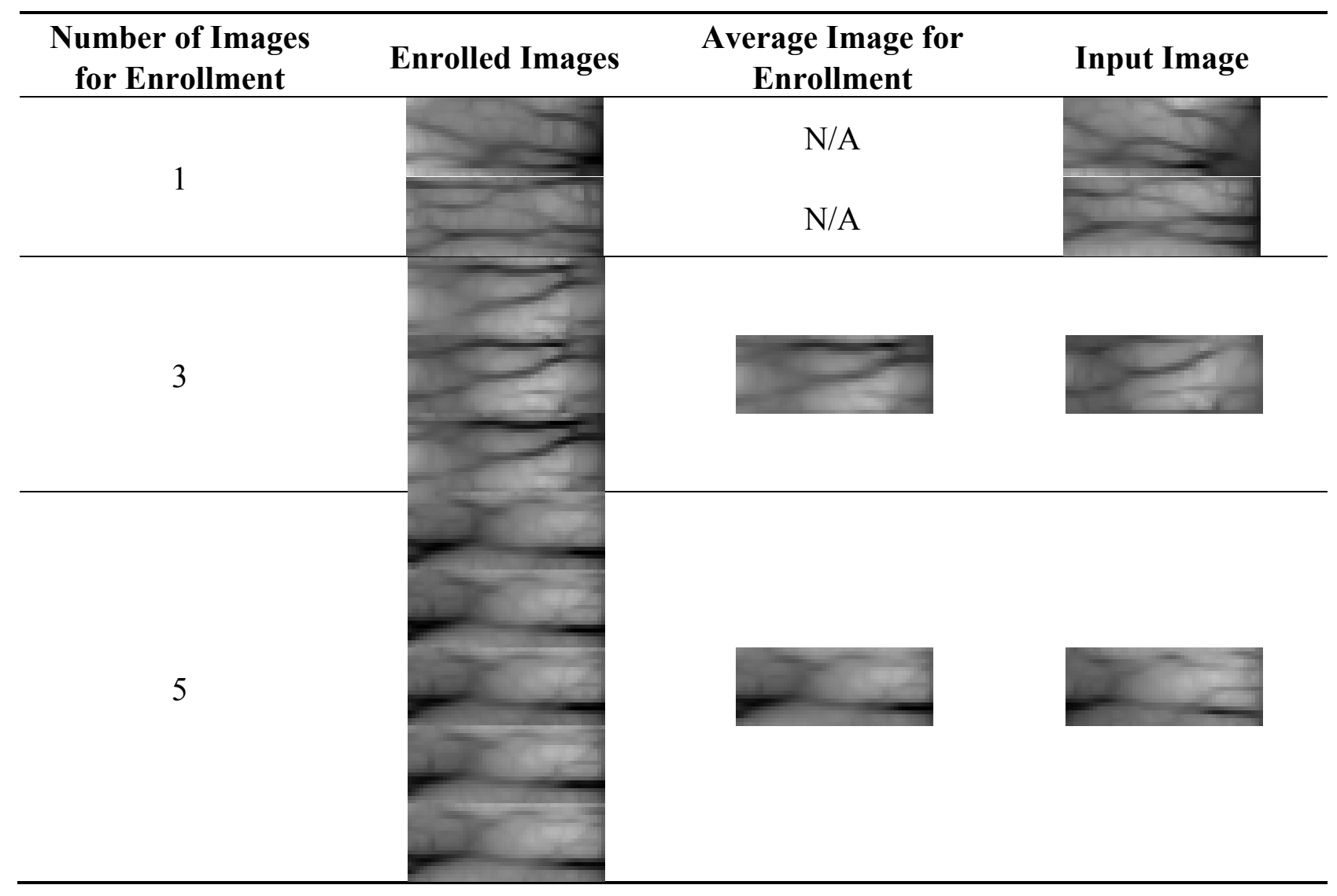

From Table 5, we can see that, when one image was used for finger-vein enrollment, false rejection was mostly caused by translational errors between images of the same finger. These errors can either occur during translations in the horizontal direction of the image (the first row of Table 5) or in the vertical direction of the image (the second row of Table 5).

The reason why false rejections occur when either three or five images are used for enrollment is as follows. The misalignment between finger-vein images selected for enrollment resulted in blurred vein lines and the appearance of artifacts in the average images that were generated. Consequently, this led to high matching distances between the enrolled finger-vein data and test data, and these cases were misclassified into the imposter matching class.

\subsection{Experimental Results Using Score-level Fusion Methods with Multiple Input Images}

In the final set of experiments, we evaluated the matching accuracies and classifying ability of the system by using score-level fusion methods with multiple input images. This involved the application of SUM and PRODUCT rules, of which the formulas are expressed by Equations (8) and (9), to combine either three or five matching scores, which were then used to classify images as being either authentic or those of an imposter.

$$
\text { SUM rule: } \quad d_{S}=\sum_{i=1}^{N} d_{i}
$$




$$
\text { PRODUCT rule: } \quad d_{P}=\prod_{i=1}^{N} d_{i}
$$

where $d_{i}$ is the original matching score between the $i$ th input finger-vein image and the one that was enrolled, and $d_{S}$ and $d P$ are the resulting matching scores obtained by using the SUM and PRODUCT rules, respectively. $N$ ( 3 or 5 ) is the number of scores to be combined.

The experiments were conducted on the good-quality database of Figure 9a as follows. From the 10 finger-vein images of each finger of an individual person in the database, we selected either three or five images as the authentic test images and used the remaining seven or five images as the enrolled images, respectively. For the imposter tests, we considered each of the 10 images of the other fingers in the database as the enrolled finger-vein image. For each enrolled image, we calculated the matching scores with the test images, combined these scores using the SUM and PRODUCT rules, and used the fused scores for final decisions. The use of this experimental method produced the same number of authentic and imposter-matching test results as for the experiments in which multiple images were used for enrollment in Section 3.3. That is because the number of images used for enrollment in the previous experiments (Section 3.3) and the number of scores used for score-level fusion in these experiments were the same, i.e., three and five. Therefore, for each of the rules, SUM and PRODUCT, when three scores were used for fusion purposes, the numbers of authentic and imposter tests were 100,800 and 17,136,000, respectively, whereas the use of the five-score-level fusion method produced 151,200 authentic matches and 35,985,600 imposter matches.

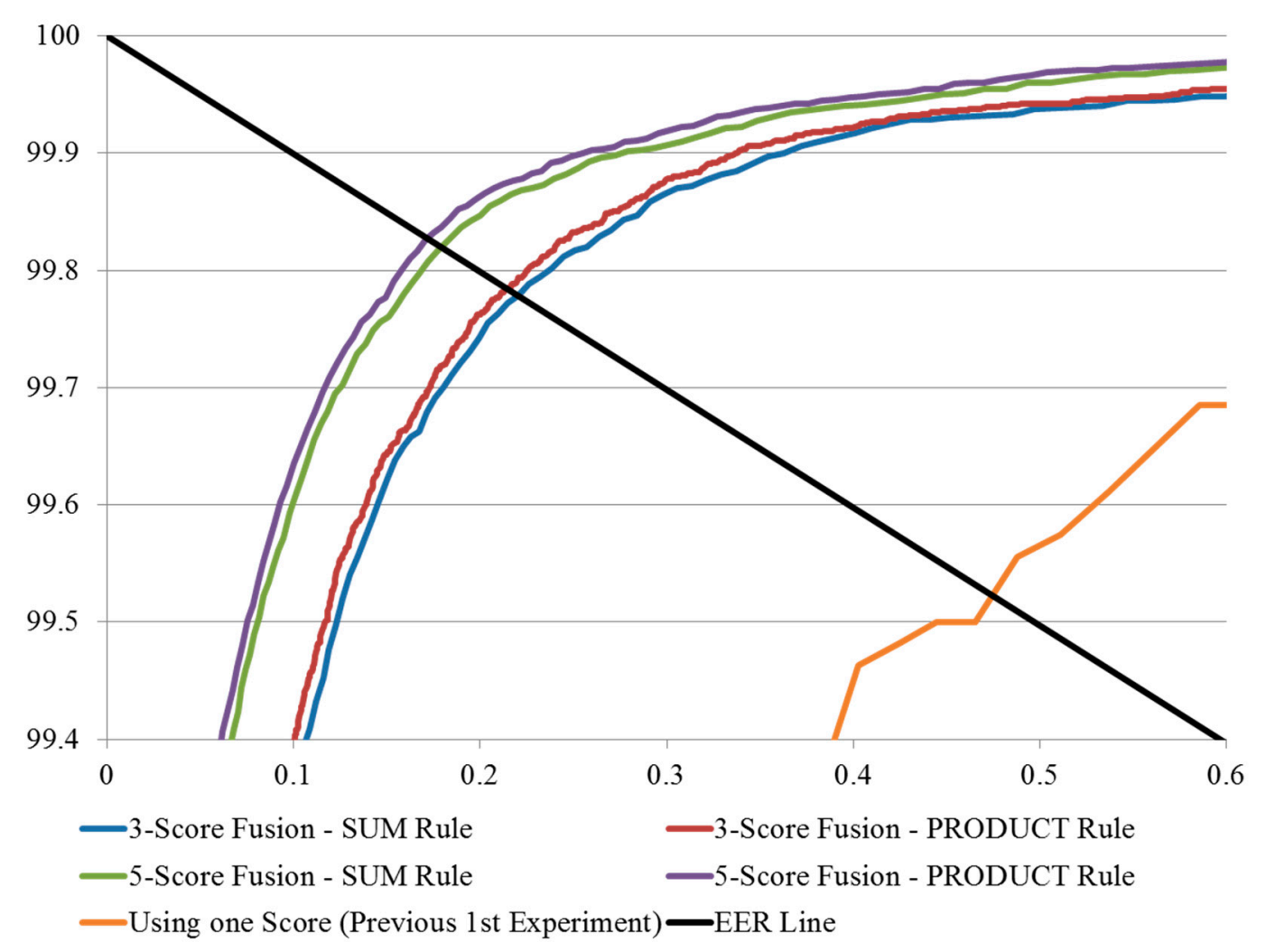

Figure 20. ROC curves obtained from the experiments with or without score-level fusion (the 1st experiment of Table 3 ) on good-quality database. 


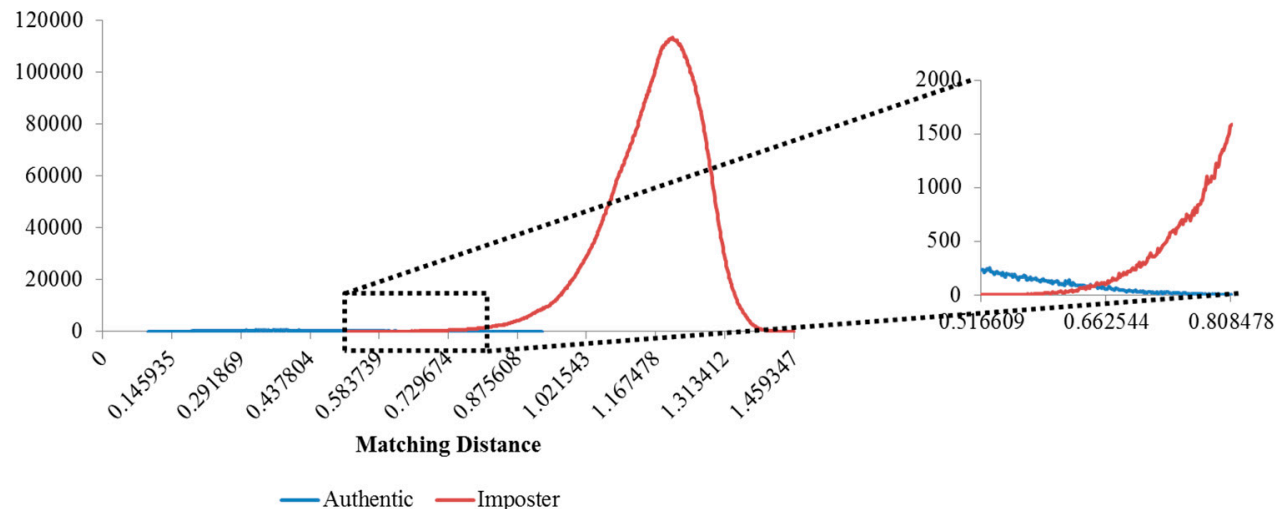

(a)

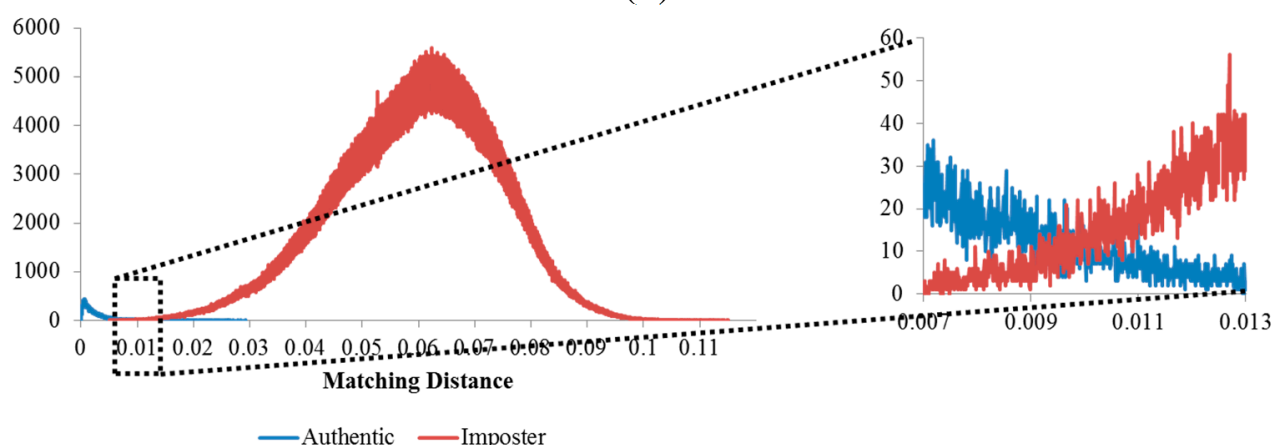

(b)

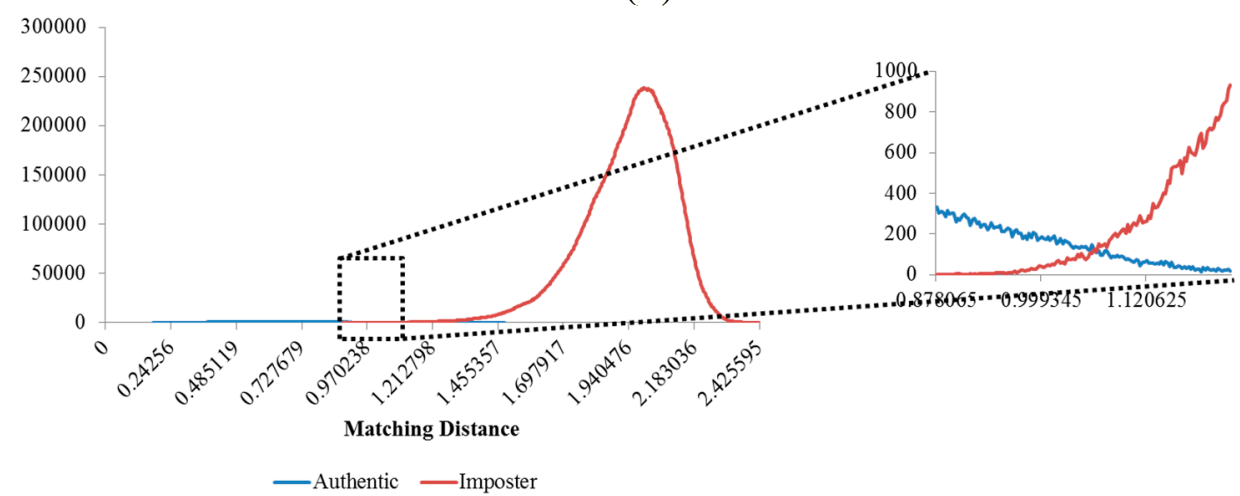

(c)

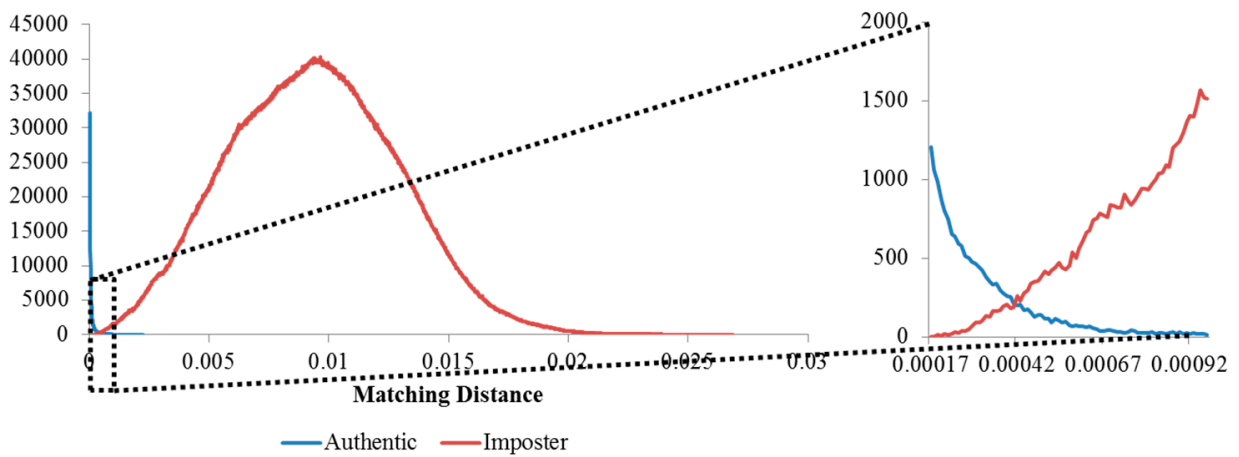

(d)

Figure 21. Matching distance distributions of authentic and imposter tests using score-level fusion methods: Three-score fusion method (a) using the SUM rule; and (b) using the PRODUCT rule; five-score fusion method (c) using the SUM rule; and (d) using the PRODUCT rule. 
The experimental results of the score-level fusion methods were compared with the previous experiment in which one score was used for finger-vein recognition (the 1st experiment performed on the good-quality database of Table 3 in Section 3.2) and shown in Table 6. The ROC curves and the distribution plots of the authentic and imposter matching scores obtained from these experiments are shown in Figures 20 and 21.

Table 6. Comparisons of the matching accuracies of the score-level fusion methods and the previous matching method in which one finger-vein image was used.

\begin{tabular}{cccc}
\hline Number of Score & Score-Level Fusion Rule & EER (\%) & d-Prime \\
\hline 1 & N/A & 0.474 & 5.805 \\
\hline \multirow{2}{*}{3} & SUM & 0.220 & 6.892 \\
& PRODUCT & 0.215 & 5.573 \\
\hline \multirow{2}{*}{5} & SUM & 0.180 & 7.183 \\
& PRODUCT & 0.172 & 3.755 \\
\hline
\end{tabular}

It can be seen from Table 6 that the score-level fusion methods enhanced the matching accuracies in that they resulted in low EER values and that the best results (the EER of $0.172 \%$ ) were obtained in case that five matching scores were fused with the PRODUCT rule. In the case of using the same number of fused scores, the PRODUCT rule produced a lower EER value compared to the SUM rule. However the d-prime value of PRODUCT rule was lower than that of SUM rule. In general, the case of lower EER produces that of the higher d-prime value only in the case that the authentic and imposter distributions are similar to Gaussian shape, respectively. However, the authentic distributions obtained by PRODUCT rule of Figure 21b,d are different from the Gaussian shape, which causes the d-prime value not to correctly reflect the accuracy of finger-vein recognition. Therefore, the d-prime value of PRODUCT rule was inconsistently lower than that of SUM rule in Table 6.

\subsection{Discussions}

Regarding the issue of using average images for feature extraction as shown in Figures 16 and 17, the method of selecting one enrolled image (whose finger-vein code shows the minimum distances compared to the codes of other enrolled images) has been most widely used (1st method). However, the finger-vein code of one image among three or five enrolled images for enrollment is selected by this method, which cannot fully compensate for the differences among three or five enrolled images. Therefore, we adopt the method of using average image for enrollment as shown in Figures 16 and 17 (2nd method). To prove this, we compared the accuracy of finger-vein recognition by this 1 st method with that by the 2 nd method. The EER (d-prime) by the 1st method with three and five images for enrollment are $0.468 \%(6.128)$ and $0.412 \%$ (6.597), respectively. By comparing the EER (d-prime) by the 2 nd method as shown in the $3 \mathrm{rd}$ and 4 th rows of Table 4, we confirm that our 2 nd method using average image for enrollment outperforms the 1 st method.

The method of simply averaging the images for enrollment can be sensitive to image alignment and detailed features can be lost in the average image. In order to solve this problem, in our research, the misalignment among the images was firstly compensated by template matching before obtaining the average image. For example in Table 5, in the case that the number of images for enrollment is 3 , 
the horizontal and vertical movements of the second enrolled image based on the first one are measured by template matching with the first enrolled image. If the measured horizontal and vertical movements of the second enrolled image are -2 and -1 pixels, respectively, for example, the compensated image is obtained by moving the original second enrolled image by +2 and +1 pixels, respectively, in the horizontal and vertical directions. From this, we can obtain the (compensated) second enrolled image where the misalignment based on the first enrolled image is minimized. Same procedure is iterated with the third enrolled image. From this procedure, we can obtain three (compensated) enrolled images where the misalignment between each other is minimized, and these three images are averaged for obtaining one enrolled image. Therefore, we can solve the problem that the average image is sensitive to image alignment and detailed features can be lost in the average image.

The total number of images in the good-quality database was 1200 (20 people $\times 2$ hands $\times 3$ fingers $\times 10$ images), and that in the mid-quality database is 1980 ( 33 people $\times 2$ hands $\times 3$ fingers $\times 10$ images). In order to obtain the meaningful conclusions and prove our conclusion irrespective of kinds of database, we also include the third open database for experiments. The total number of images in the open database was 3816 (106 people $\times 2$ hands $\times 3$ fingers $\times 6$ images). Consequently, a total of 6996 images were used for our experiments, and we obtained the conclusion through a great deal of authentic and imposter matching, as shown in Table 2.

The original LBP used in our method can be more sensitive to noise than the uniform LBP. Therefore, in our method, the sub-sampled image of $50 \times 20$ pixel is used for feature extraction by LBP as shown in Figure 4c,f,i, which can reduce the noise in the image for feature extraction. In addition, the two cases of LBP codes in Figure 22c are assigned as same decimal code of 1 by the uniform LBP although they are actually different LBP code (00000001 (left case) and 00010000 (right case)), which can reduce the dissimilarity between two different patterns of finger-vein image. Therefore, we use the original LBP method in our research.

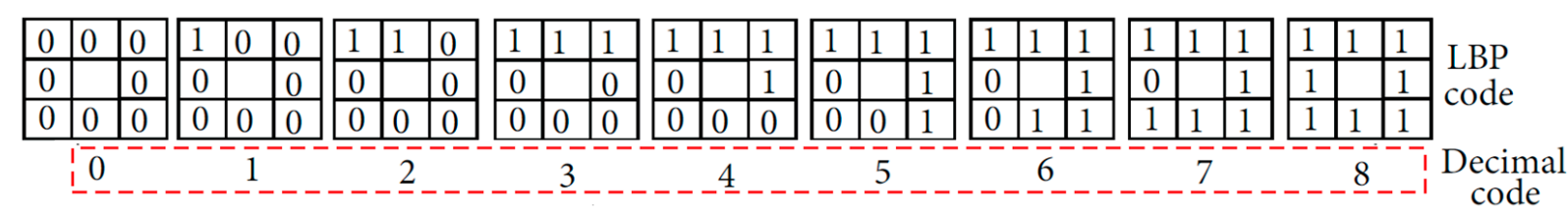

(a)

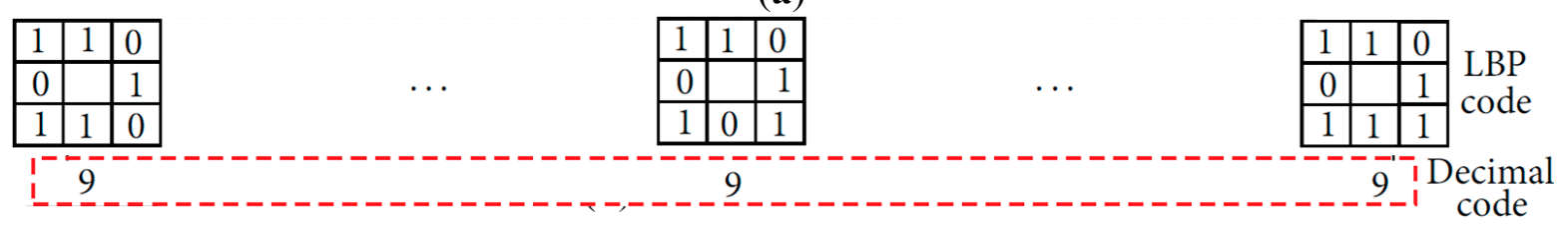

(b)

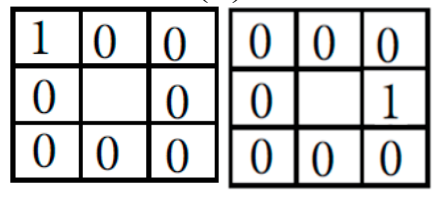

(c)

Figure 22. Example of uniform and nonuniform patterns and their assigned decimal codes by uniform LBP, respectively: (a) uniform patterns; (b) nonuniform patterns; (c) two cases of decimal code of 1 by uniform LBP. 
We compared the accuracies by our original LBP and those by uniform LBP. The EER (d-prime) by uniform LBP with 1st, 2nd, and 3rd databases are $0.925 \%$ (5.122), 4.513\% (3.443), and $12.489 \%$ (2.114). The EERs by uniform LBP are larger than those by original LBP of the 1st row of Table 3. In addition, the d-prime values by uniform LBP are smaller than those by original LBP of the 1st row of Table 3. From that, we can confirm that the performance by our original LBP is better than that by uniform LBP.

\section{Conclusions}

This paper proposed a new finger-vein capturing device that relies on accurate finger positioning to reduce misalignment when vein images are captured. This device was used to capture images to construct a database composed of good-quality finger-vein images, which were compared to the images in the mid-quality database (which was used in previous research) and an open database. The images in the good-quality database produced lower matching EER and higher d-prime values. Based on the comparative experimental results considering finger-vein dissimilarities between people, hands, and fingers in the three databases, we evaluated the factors that affect the accuracy of finger-vein recognition and concluded that finger-vein dissimilarity decreases for people, fingers, and hands in that order. We also proposed a method based on the use of multiple images to generate an image for finger-vein enrollment, instead of using one image as done previously. For our final set of experiments, we proposed a recognition method using score-level fusion obtained by using SUM and PRODUCT rules. The experimental results obtained for images from the database captured by our device, showed that the use of multiple enrollment images and score-level fusion could enhance the matching accuracies by reducing the EER. For future work, we plan to evaluate the various factors determining the accuracies of hand vein or palm vein recognition systems. In addition, we would also consider evaluating the effect of race, age, and gender on the accuracy of vein recognition.

\section{Acknowledgments}

This research was supported by the Basic Science Research Program through the National Research Foundation of Korea (NRF) funded by the Ministry of Education (NRF-2012R1A1A2038666).

\section{Author Contributions}

Tuyen Danh Pham and Kang Ryoung Park designed the overall system for finger-vein recognition. In addition, they wrote and revised the paper. Young Ho Park, Dat Tien Nguyen, and Seung Yong Kwon implemented the algorithm of score-level fusion, and helped with the collecting database with the analyses of experimental results.

\section{Conflicts of Interest}

The authors declare no conflict of interest.

\section{References}

1. Prabhakar, S.; Pankanti, S.; Jain, A.K. Biometric recognition: Security and privacy concerns. IEEE Secur. Priv. 2003, 1, 33-42. 
2. Turk, M.A.; Pentland, A.P. Face Recognition Using Eigenfaces. In Proceedings of the IEEE Conference on Computer Vision and Pattern Recognition, Maui, HI, USA, 3-6 June 1991; pp. 586-591.

3. Daugman, J. How iris recognition works. IEEE Trans. Circuits Syst. Video Technol. 2004, 14, 21-30.

4. Jain, A.K.; Ross, A.; Prabhakar, S. An introduction to biometric recognition. IEEE Trans. Circuits Syst. Video Technol. 2004, 14, 4-20.

5. Yang, W.; Hu, J.; Wang, S. A finger-vein based cancellable bio-cryptosystem. Lect. Notes Comput. Sci. 2013, 7873, 784-790.

6. Yang, J.F.; Yang, J.L. Multi-Channel Gabor Filter Design for Finger-Vein Image Enhancement. In Proceedings of the Fifth International Conference on Image and Graphics, Xi'an, China, 20-23 September 2009; pp. 87-91.

7. Shin, K.Y.; Park, Y.H.; Nguyen, D.T.; Park, K.R. Finger-vein image enhancement using a fuzzy-based fusion method with Gabor and Retinex filtering. Sensors 2014, 14, 3095-3129.

8. Park, Y.H.; Park, K.R. Image quality enhancement using the direction and thickness of vein lines for finger-vein recognition. Int. J. Adv. Robot. Syst. 2012, 9, 1-10.

9. Yang, J.; Shi, Y. Finger-vein ROI localization and vein ridge enhancement. Pattern Recognit. Lett. 2012, 33, 1569-1579.

10. Yang, J.; Wang, J. Finger-Vein Image Restoration Considering Skin Layer Structure. In Proceedings of the International Conference on Hand-Based Biometrics, Hong Kong, China, 17-18 November 2011; pp. 1-5.

11. Yang, J.; Zhang, B.; Shi, Y. Scattering removal for finger-vein image restoration. Sensors 2012, 12, 3627-3640.

12. Cho, S.R.; Park, Y.H.; Nam, G.P.; Shin, K.Y.; Lee, H.C.; Park, K.R.; Kim, S.M.; Kim, H.C. Enhancement of finger-vein image by vein line tracking and adaptive gabor filtering for finger-vein recognition. Appl. Mech. Mater. 2011, 145, 219-223.

13. Zhang, J.; Yang, J. Finger-Vein Image Enhancement Based on Combination of Gray-Level Grouping and Circular Gabor Filter. In Proceedings of the International Conference on Information Engineering and Computer Science, Wuhan, China, 19-20 December 2009; pp. 1-4.

14. Pi, W.; Shin, J.; Park, D. An Effective Quality Improvement Approach for Low Quality Finger Vein Image. In Proceedings of the International Conference on Electronics and Information Engineering, Kyoto, Japan, 1-3 August 2010; pp. V1-424-V1-427.

15. Yu, C.B.; Zhang, D.M.; Li, H.B.; Zhang, F.F. Finger-Vein Image Enhancement Based on Multi-Threshold Fuzzy Algorithm. In Proceedings of the International Congress on Image and Signal Processing, Tianjin, China, 17-19 October 2009; pp. 1-3.

16. Yang, J.; Zhang, X. Feature-level Fusion of Global and Local Features for Finger-Vein Recognition. In Proceedings of IEEE 10th International Conference on Signal Processing, Beijing, China, 24-28 October 2010; pp. 1702-1705.

17. Lu, Y.; Yoon, S.; Park, D.S. Finger vein recognition based on matching score-level fusion of gabor features. J. Korean. Inst. Commun. Inf. Sci. 2013, 38A, 174-182.

18. Qian, X.; Guo, S.; Li, X.; Zhong, F.; Shao, X. Finger-vein Recognition Based on the Score Level Moment Invariants Fusion. In Proceedings of International Conference on Computational Intelligence and Software Engineering, Wuhan, China, 11-13 December 2009; pp. 1-4. 
19. Yang, G.; Xi, X.; Yin, Y. Finger vein recognition based on a personalized best bit map. Sensors 2012, 12, 1738-1757.

20. Park, Y.H.; Tien, D.N.; Lee, H.C.; Park, K.R.; Lee, E.C.; Kim, S.M.; Kim, H.C. A Multimodal Biometric Recognition of Touched Fingerprint and Finger-Vein. In Proceedings of International Conference on Multimedia and Signal Processing, Guillin, China, 14-15 May 2011, pp. 247-250.

21. Nguyen, D.T.; Park, Y.H.; Lee, H.C.; Shin, K.Y.; Kang, B.J.; Park, K.R. Combining touched fingerprint and finger-vein of a finger, and its usability evaluation. Adv. Sci. Lett. 2012, 5, 85-95.

22. Cui, F.; Yang, G. Score level fusion of fingerprint and finger vein recognition. J. Comput. Inf. Syst. 2011, 7, 5723-5731.

23. He, M.; Horng, S.J.; Fan, P.; Run, R.S.; Chen, R.J.; Lai, J.L.; Khan, M.K.; Sentosa, K.O. Performance evaluation of score level fusion in multimodal biometric systems. Pattern Recognit. 2010, 43, 1789-1800.

24. Qin, B.; Pan, J.-F.; Cao, G.-Z.; Du, G.-G. The Anti-spoofing Study of Vein Identification System. In Proceedings of International Conference on Computational Intelligence and Security, Beijing, China, 11-14 December 2009; pp. 357-360.

25. Nguyen, D.T.; Park, Y.H.; Shin, K.Y.; Kwon, S.Y.; Lee, H.C.; Park, K.R. Fake finger-vein image detection based on fourier and wavelet transforms. Digit. Signal Process. 2013, 23, 1401-1413.

26. Webcam C600. Available online: http://www.logitech.com/en-us/support/5869 (accessed on 15 April 2015).

27. Dongguk Finger-Vein Database. Available online: http://dm.dgu.edu/link.html (accessed on 4 June 2015).

28. SDUMLA-HMT Finger Vein Image Database. Available online: http://mla.sdu.edu.cn/sdumla-hmt.html (accessed on 15 April 2015).

29. Yin, Y.; Liu, L.; Sun, X. SDUMLA-HMT: A multimodal biometric database. Lect. Notes Comp. Sci. 2011, 7098, 260-268.

(C) 2015 by the authors; licensee MDPI, Basel, Switzerland. This article is an open access article distributed under the terms and conditions of the Creative Commons Attribution license (http://creativecommons.org/licenses/by/4.0/). 